\title{
An evolutionary Cournot model with limited market knowledge
}

\author{
Gian Italo Bischi ${ }^{\mathrm{a}, *}$, Fabio Lamantia ${ }^{\mathrm{b}}$, Davide Radi ${ }^{\mathrm{c}}$ \\ ${ }^{a}$ DESP, University of Urbino "Carlo Bo", Urbino (Italy) \\ ${ }^{b}$ DESF, University of Calabria, Rende (Italy) and School of Social Sciences, University of Manchester (UK) \\ ${ }^{c}$ Department of Management, Marche Polytechnic University, Ancona (Italy)
}

\begin{abstract}
In this paper we analyze a dynamic game of Cournot competition with heterogeneous firms choosing between two different adaptive behavioral rules in deciding output strategies. The underling oligopoly structure is standard: using a constant returns to scale technology, $N$ firms produce homogeneous goods, which are sold in a market characterized by constant price elasticity. In this setup, we assume that a fraction of firms employs a quite rough rule of thumb, the so-called Local Monopolistic Approximation (LMA), whereas the complementary fraction plays Best Reply (BR), a more demanding strategy in terms of information and computation requirements. The model is first considered with exogenously fixed fractions of firms in the two complementary groups. Then it is generalized by considering an endogenous evolutionary switching process between the two behavioral strategies based on profit-driven replicator dynamics. The role of the number of firms, information costs and inertia (or anchoring attitude) in production decisions is analyzed, as well as the influence in the evolutionary process of random noise in the demand function and memory of past profits. Global properties of the oligopoly with evolutionary pressure between behavioral rules are discussed, with particular regard to cases in which the Nash equilibrium is unstable.
\end{abstract}

JEL classification: C73, D83, L13.

Keywords: Oligopolies, Local Monopolistic Approximation, Evolutionary dynamics, Heterogeneous firms, Nonlinear phenomena.

\section{Introduction}

In Bischi et al., 2007 two different discrete-time Cournot models have been considered in an oligopoly with isoelastic demand, homogeneous goods and linear production costs: the classical Best Reply (BR) dynamics with naïve expectations and an alternative adjustment mechanism called Local Monopolistic Approximation (LMA). Under the latter, at each time step firms solve a profit maximization problem employing a linear extrapolation of the demand function and ignore the effects of the competitors' outputs on the selling price 1 Both adjustment mechanisms share the same Nash equilibrium as their unique steady state. In the duopoly case, Bischi et al., 2007 prove that the Nash equilibrium is always a stable equilibrium under LMA adjustments, but it becomes unstable under BR adjustments, provided that competitors have sufficiently different marginal costs: this leads to the intriguing statement that "less information implies more stability". However, for parameters values such that both models converge to the Nash equilibrium, Bischi et al., 2007 numerically show that the Nash equilibrium may have a basin of attraction that is larger under BR than under LMA. In any case, a more interesting comparison of these two models should involve profits generated over time by adopting these different adjustment rules. As argued in the conclusions of Bischi et al., 2007, this may be done by considering an explicit dynamic modeling of the two different mechanisms under evolutionary pressure, in order to investigate whether one kind of adjustment will dominate, and consequently drive out, the other one in the long run.

In this paper, we carry out this idea. For this purpose, we consider an industry with firms subdivided into two complementary fractions: a fraction of them adjusts outputs employing LMA, whereas the rest of the industry produces according to BR. These fractions change over time on the basis of the observed performance of both behavioral strategies, which is measured in terms of realized profits. Models with evolutionary updating of the fractions of agents, who adopt

\footnotetext{
* Corresponding author

Email addresses: gian.bischi@uniurb.it (Gian Italo Bischi), lamantia@unical.it (Fabio Lamantia), d.radi@univpm.it (Davide Radi) 1 Tuinstra, 2004 considers a similar setup in monopolistic competition with price adjustment for learning the demand function.
} 
different expectations formation rules, have recently been proposed by many authors in economic and financial models, see e.g. Brock and Hommes, 1997, Hommes, 2013, Droste et al., 2002, Hommes et al., 2011 and Anufriev et al., 2013. In particular, Droste et al., 2002 consider an evolutionary Cournot duopoly with homogeneous goods, linear demand and quadratic production costs. Pairs of firms, each with its own behavioral rule, are randomly matched at every time period to play the game. The authors show that when firms can select between a cheap BR rule and a costly 'Nash' rule, endogenous fluctuations and complicated dynamics may arise, mainly due to the dominance of BR behavior in a neighborhood of the Nash equilibrium because of information costs. Hommes et al., 2011 consider a similar evolutionary setup with linear demand and linear production costs but with random matching of $\mathrm{N}$ firms at a time, which can switch, on the basis of past performances, between costly rational and cheap boundedly rational expectation rules on aggregate output of their rivals. In this case, Hommes et al. 2011 find that the classic Theocharis' result on the instability of the Nash equilibrium with more than three firms is also confirmed.

In our case, all the agents are boundedly rational. However, they use different adaptive behavioral rules involving different degrees of information and different computational effort: BR firms know the true demand function and solve a nonlinear optimization problem to compute next period outputs, whereas LMA firms base their decisions on the simpler 'rule of thumb' described above, which involves a lower amount of information and simpler computations. In other words, the heterogeneity among agents concerns the information set that they employ. The mechanism of evolutionary selection introduced in the model states that according to an observable fitness measure (or index of performance) agents who adopt LMA may decide to switch to the BR behavior, or, vice-versa, BR agents may decide to switch to the less sophisticated LMA behavior. The latter may appear strange, as it implies that agents prefer to ignore their information about the demand function and use a linear approximation instead. However, this is consistent with the profit-driven evolutionary process if these agents realize that they could be better off by using less information 2

In this paper, we first propose a two-dimensional dynamic Cournot model with heterogeneous agents. A population of $N$ firms is subdivided into two (fixed) fractions $r$ and $(1-r), r \in[0,1]$, that adopt BR and LMA behavioral rules respectively. For this model, the dynamic variables are the quantities played by a representative agent from the BR and the LMA group. Agents play an oligopoly game with undifferentiated goods against the average aggregate production. The stability of the (unique) Nash equilibrium as the number $N$ of firms increases is analyzed, a classical problem dating back to the work of Theocharis, 1960 (see also Fisher, 1961, Hahn, 1962, McManus and Quandt, 1961 and Hommes et al., 2011). In addition, the influence on equilibrium stability of inertia in revising productions and of the size of groups with different adaptive rules is addressed.

Then we endogenize the dynamics of the fraction of firms playing the behavioral rules, according to profit-driven evolutionary pressure. The switching mechanism considered here is an improvement of the standard replicator dynamics that was first proposed in Cabrales and Sobel, 1992 (see also Hofbauer and Sigmund, 2003, and Hofbauer and Weibull, 1996) and takes a monotone transformation of a discounted average of past profits as fitness measure. The resulting mathematical model is a four-dimensional dynamical system. We first address the case in which the fitness measure only depends on last period profits. In this case, the mathematical model reduces to a three-dimensional discrete dynamical system, where the first two variables represent quantity dynamics as before and the third dynamic variable expresses the time evolution of the fraction of firms following one behavioral rule. In this three-dimensional dynamic model, the influence of information costs on the stability of the Nash equilibrium is analytically studied. However, interesting situations are observed with asymptotic dynamics not converging to the Nash equilibrium. Indeed, time evolutions towards the invariant planes $r=0$ and $r=1$, where all players follow the same behavioral rule (pure LMA or pure BR oligopoly) are numerically analyzed, as well as the crucial role of initial conditions. The dynamics on the invariant planes $r=0$ and $r=1$ are governed by two-dimensional restrictions of the model that assume the form of triangular maps (see Kolyada, 1992 and Dieci et al., 2001), so that many properties of the attractors can be deduced analytically. Moreover, several other long-run evolutions are numerically detected, characterized either by the coexistence in the long run of both LMA and BR behaviors, i.e. cyclic or chaotic attractors involving intermediate values of $r$, or by possibly on-off intermittency phenomena.

The inclusion of a discounted average of past profits (a 'memory effect') in the evolutionary process is then encompassed, thus leading to study the complete four-dimensional dynamical system, which maintains dynamical properties that are similar to those without memory. Finally, the introduction of random noise in the demand function is examined through numerical computations, in order to check whether the results obtained in a deterministic setting are robust in the presence of some randomness.

\footnotetext{
${ }^{2}$ This may be seen as a form of Occam's razor (or 'law of parsimony') stating that among competing behaviors that perform equally well, the simpler one should be selected.
} 
Given the above described setup, the main research questions we address in our work can be summarized as follows: (1) does a behavioral rule using more information and computational efforts always perform better than a less rational one when information costs are negligible? (2) What is the relationship between stability of the Nash equilibrium in the parameters space and stability of the Nash equilibrium in the state space when BR and LMA agents interact? (3) What is the most likely long-run industry outcome when firms can switch between different (boundedly rational) behavioral rules but they fail to converge to a Nash equilibrium? (4) Can behavioral heterogeneity arise endogenously as a result of market interaction among ex-ante identical agents who can select between different behavioral rules? (5) Are these results influenced by the presence of random noise in market demand? (6) What is the role of memory in the system, with agents employing a discounted average of past profit as index of performance in their strategic decisions?

The outline for the rest of this paper is as follows. In Section 2 we consider the two-dimensional dynamical system that represents the time evolution of quantities in an oligopoly with nonlinear demand and linear production costs with firms subdivided into two fixed complementary fractions that adopt BR and LMA behavioral strategies. In Section 3 we enhance the model by endogenizing the time evolution of the fraction of firms playing BR through profit driven evolutionary pressure. Memory of past profits in the fitness measure is also included in the system. The dynamics of this model is then studied in Section 4, where several insights on global dynamic properties of the model are provided. In this Section, we also address the presence of random noise in market demand and discuss the role of memory in the fitness measure. Section 5 concludes.

\section{The oligopoly game with fixed fractions of heterogeneous agents}

Consider an industry with $N \geq 2$ firms indexed by $i=1, \ldots N$, producing homogeneous goods with linear cost of the form

$$
C_{i}(t)=c q_{i}(t)+K_{i}
$$

where $q_{i}(t)$ is the quantity produced by firm $i$ at time $t, c>0$ denotes its marginal cost (we consider the case of firms with the same production technology ${ }^{3}$ ) and $K_{i}$ represents the information and computation costs of firm $i$, whose purpose is specified below. All firms operate in a market characterized by an isoelastic demand function

$$
p(t)=f(Q(t))=\frac{A(t)}{Q(t)}
$$

where $Q(t)=\sum_{i=1}^{N} q_{i}(t)$ is the total output of the industry at time $t$ and $A(t) \sim \mathcal{U}[1-\epsilon, 1+\epsilon]$, with $1>\epsilon \geq 0$, is an IID random variable with uniform distribution that represents the aggregate consumers' expenditures at time $t 5^{5}$ Production decisions are assumed to occur at discrete time periods $t=0,1,2 \ldots$ (decision driven time). Let $r \in[0,1]$ be the fraction of firms that decide their next period output according to Best Reply (BR) with naïve expectations, and the complementary fraction $(1-r)$ decides according to Local Monopolistic Approximation (LMA). In the following we denote by $x(t)$ the output at time $t$ of the representative firm of the first group, say BR firm, and by $y(t)$ the output at time $t$ of the representative firm of the second group, say LMA firm. With these notations, the total output at time $t$ can be written as

$$
Q(t)=N[r x(t)+(1-r) y(t)]
$$

In the following we focus on dynamics such that $Q(t)>0 \forall t \in \mathbb{N}$, which implies either $x(t)>0$ or $y(t)>0, \forall t \in \mathbb{N}$. We indicate with $D$ the set of initial conditions such that the game satisfies this positive aggregate output condition.

\footnotetext{
${ }^{3}$ This assumption is motivated by the fact that in the second part of the paper the fraction of players of the two types is endogenized, so that firms are ex-ante homogeneous with respect to the production technology and they only differ in the behavioral rule (BR or LMA) for deciding future production.

${ }^{4}$ This inverse demand function can be derived by assuming that a representative consumer is endowed with a log-linear (or Cobb-Douglas) utility function, as recalled in Lambertini, 2010 In this paper we have considered this particular specification, with elasticity constantly equal to one, for the sake of comparison with the literature, starting from Puu, 1991 (see also Bischi et al. 2010, Tramontana et al. 2010, Agliari et al. 2006 and Lamantia 2011), and for easiness of computation. With respect to the latter point, Agliari and Puu 2002 study a duopoly with bounded isoelastic demand, obtaining essentially the same results as in the (easier) version with unbounded demand, which is considered here.

${ }^{5}$ In this paper the dynamic analysis and main results are provided for a constant expenditure equal to $\mathbb{E}[A(t)]$, which is equivalent to impose $\epsilon=0$. In the last part of the paper, a random $A(t)$ is considered to validate the deterministic results in a more general stochastic setting.
} 
The adjustment mechanism by which the representative BR firm with naïve expectations updates its production is expressed by

$$
\begin{aligned}
x(t+1) & =\max \left\{0,(1-\lambda) x(t)+\lambda R\left(Q_{-1}(t)\right)\right\}= \\
& =\max \left\{0,(1-\lambda) x(t)+\lambda\left[\sqrt{\frac{A(t) Q_{-1}(t)}{c}}-Q_{-1}(t)\right]\right\}
\end{aligned}
$$

where $R($.$) is the reaction function (see \mathrm{Puu}, 1991$ and Bischi et al., 2010 for details) and $\lambda \in(0,1]$ is an adaptive adjustment parameter, also named speed of adjustment, that represents the degree of confidence that each firm has about the Cournotian BR behavioral assumption ${ }^{6}$ The quantity $Q_{-1}(t)$ is the average production of the rest of the industry at time $t$, equal for all BR players and defined as

$$
Q_{-1}(t)=(N-1)[r x(t)+(1-r) y(t)]
$$

Under naïve expectations, $Q_{-1}(t)$ can be employed as a proxy for $Q_{-1}^{e}(t+1)$, the expected production of the rest of the industry for time $t+1$ (see also Droste et al. 2002 for the same assumption).

Now consider a representative LMA firm (see Bischi et al., 2007 for details), which adjusts its production according to the rule

$$
y(t+1)=\max \left\{0,(1-\alpha) y(t)+\frac{\alpha}{2}\left[y(t)+\frac{c-f(Q(t))}{f^{\prime}(Q(t))}\right]\right\}
$$

where $f(Q(t))$ is given by (2) with [3], $f^{\prime}(Q(t))=-\frac{A(t)}{(Q(t))^{2}}$ and $\alpha \in(0,1]$ is an adaptive adjustment parameter, also named speed of adjustment, that expresses the degree of confidence that each firm has about the LMA behavioral assumption 7 The max operators in (4) and in (6) impose nonnegativity of productions whenever BR or LMA with inertia return negative quantities; in these cases, firms simply decide not to produce for the next time period.

Assuming $\epsilon=0$, the two difference equations (4) and (6) form a two-dimensional dynamical system that describes the time evolution of a Cournot oligopoly with fixed fractions of heterogeneous agents, which can be rewritten as follow: 8

$$
\left\{\begin{array}{l}
x(t+1)=\max \left\{0,(1-\lambda) x(t)+\lambda\left[\sqrt{\frac{(N-1)[r x(t)+(1-r) y(t)]}{c}}-(N-1)(r x(t)+(1-r) y(t))\right]\right\} \\
y(t+1)=\max \left\{0,(1-\alpha) y(t)+\frac{\alpha}{2}[y(t)+N(r x(t)+(1-r) y(t))(1-c N(r x(t)+(1-r) y(t)))]\right\}
\end{array}\right.
$$

with parameters $N \geq 2, r \in[0,1], \alpha \in(0,1]$ and $\lambda \in(0,1]$ in addition to the marginal cost $c>0.9$

At this point, it is useful to sum up similarities and differences for BR and LMA players in terms of minimal information and computational efforts. At each time $t$, agents of either type know their own current output, their own production cost and observe the current selling price $p(t)$. On the one hand, the generic BR agent: (1a) makes use of the knowledge of the inverse demand function in (2); (2a) has naïve expectations on the quantity of the rest of the industry, according to (5); (3a) solves an optimization problem, which involves solving a nonlinear equation. On the other hand, the generic LMA agent: (1b) does not employ knowledge of the inverse demand function (2), but only assesses its linear approximation with respect to its own production ${ }^{10}(2 \mathrm{~b})$ does not form expectations on other firms' future productions when deciding its own production: an LMA agent neglects the effects of strategic interaction among players' decisions; (3b) solves a quadratic optimization problem, which involves solving a linear equation ${ }^{11}$.

Notice that in any case a LMA agent always needs less information and computational skills to determine its next period output. For these reasons, using a slight abuse of language aimed to emphasize the differences, we can refer to the strategy followed by LMA agents as the strategy 'using less information'.

\footnotetext{
${ }^{6}$ Notice that (4) reduces to the standard BR dynamics for $\lambda=1$, see again Bischi et al. 2010. Moreover, $1-\lambda$ expresses inertia (or anchoring) of the representative BR player.

${ }^{7}$ Observe that 6 reduces to the standard LMA dynamics for $\alpha=1$. Similarly to $1-\lambda$ for the BR dynamics, $1-\alpha$ represents the possible inertia of the representative LMA player.

${ }^{8}$ The presence of the random parameter $A(t)$ is neglected in the oligopoly model with fixed $r$ as it is not relevant to the aim of the present investigation. However, it will be considered in the next Sections when the evolutionary version of this oligopoly is introduced and studied.

${ }^{9}$ Notice that in the Cournot oligopoly $(7)$, at some periods of time firms using either the BR heuristic or the LMA heuristic may find convenient to halt their production, i.e. either $x(t)=0$ or $y(t)=0$. This does not prevent that in the next period firms decide to provide positive quantities and the possibility to converge to an equilibrium with positive productions in the long run (see, e.g. Fanti et al. 2013 for a similar issue).

${ }^{10}$ See Bischi et al. 2007 for a discussion on how this information can be empirically obtained.

${ }^{11}$ This last sentence holds when production costs are linear (as in this paper) or quadratic. See Bischi et al. 2007 for details.
} 
The dynamical system (7) represents an oligopoly game with both BR and LMA firms that has not been proposed before and constitutes an helpful starting point to understand the dynamics of the more sophisticated evolutionary oligopoly game presented later in Section 3. Moreover, the dynamics of this simplified model offer interesting and economically insightful results such as the conditions for the existence and stability of production equilibria. The details are contained in the following:

Proposition 1. Dynamical system (7) admits the (unique) Nash equilibrium:

$$
E_{*}=\left(\frac{N-1}{c N^{2}}, \frac{N-1}{c N^{2}}\right)
$$

Moreover,

- if $N \in\{2,3\}$, then $E_{*}$ is a stable node for all parameters values;

- if $N=4$, then $E_{*}$ is a stable node for all $r \in[0,1]$ and $\lambda \in(0,1)$; in the case $\lambda=1$ (classical BR), stability of $E_{*}$ holds for $r \in[0,1)$;

- if $5 \leq N<N_{f}$, with

$$
N_{f}=\frac{4[4+\alpha(1-2 r)]-2 \lambda(1-r)(4+\alpha)}{\alpha(4+r(\lambda-4)-2 \lambda)+4 r \lambda}
$$

then $E_{*}$ is a stable node.

- In addition, $E_{*}$ loses stability for $N$ increasing beyond $N_{f}$ through a period-doubling bifurcation.

Proof. See the Appendix.

Proposition 1 confirms qualitatively for this oligopoly game the Theocharis' result in Theocharis, 1960 on the destabilizing role of the number of players $N$. However, for our purposes the main result from Proposition 1 concerns the influence of parameters $r \in[0,1], \lambda \in(0,1]$ and $\alpha \in(0,1]$ on the range of stability of the Nash equilibrium $E_{*}$. In fact, from

$$
\frac{d N_{f}}{d r}=\frac{2(4-\alpha)(\lambda-2)(4 \lambda+\alpha \lambda-4 \alpha)}{(\alpha(4+r(\lambda-4)-2 \lambda)+4 r \lambda)^{2}}
$$

it follows that:

Corollary 2. The bifurcation value $N_{f}$ is such that $\frac{d N_{f}}{d r}<0$ whenever $(\lambda, \alpha) \in(0,1] \times(0,1]$ is above the equilateral hyperbola $4 \lambda+\alpha \lambda-4 \alpha=0$ and it is such that $\frac{d N_{f}}{d r} \geq 0$ otherwise. In particular, under the condition $\lambda \geq \alpha$, i.e. if $B R$ players' inertia is less than or equal to LMA players' inertia, $N_{f}$ decreases in $r$. Furthermore, $N_{f}$ increases as the parameters $\alpha$ and $\lambda$ decrease. In the particular case $\alpha=\lambda=1$ (no inertia) we have $N_{f}=\frac{2 r+10}{r+2}$, so that $N_{f}=4$ for $r=1$ and $N_{f}=5$ for $r=0$.

Moreover, when $N \geq 5$ :

- Consider a fixed fraction of BR players $\bar{r} \in(0,1]$. If $\alpha=1$, then the stability of (8) can be achieved only with $\lambda \in\left(0, \frac{4}{5}\right)$. In other words, when LMA players have no inertia, stability can be obtained only if BR players have a sufficiently 'high' level of inertia;

- Consider a fixed fraction of BR players $\bar{r} \in(0,1]$. If $\lambda=1$, then the stability of (8) can be achieved only with $\alpha \in\left(0, \frac{8-4(N-2) \bar{r}}{N(2-3 \bar{r})+6 \bar{r}-2}\right)$ and $\bar{r} \in\left(0, \frac{2}{N-2}\right]$. In other words, when BR players have no inertia, stability can be obtained only if LMA players have a sufficiently 'high' level of inertia and the fraction of BR players is sufficiently 'low';

- Consider all LMA firms, i.e. $\bar{r}=0$, the condition for the stability of (8) reduces to $\alpha \in\left(0, \frac{4}{N-1}\right)$ : a sufficient level of inertia must be imposed to LMA firms in order to have stability, with more and more inertia as the number of firms in the market increases. 
From Corollary 2, it comes out that more inertia enlarges the stability range of the Nash equilibrium. Moreover, if the speed of adjustment of BR firms is higher than the speed of adjustment of LMA firms, then an increasing fraction of BR players causes a reduction of the range of stability of the Nash equilibrium. Of course the opposite effect is obtained by increasing the fraction of LMA players.

It is also worth to point out that $N_{f}$ is a monotone function of $r$, see 100 . It follows that whenever the Cournot equilibrium is stable both with all LMA firms and with all BR firms, then it is stable with any combination of the two. This underlines that heterogeneity does not have a destabilizing effect on the dynamics of oligopoly (7).

All the considerations up to now only concern the local asymptotic stability of the Nash equilibrium $E_{*}$. However, numerical simulations suggest other two interesting properties: first, whenever $E_{*}$ is locally stable, it is also globally stable in $D$; second, when $E_{*}$ loses stability due to an increase in the number $N$ of firms or, given a sufficiently high $N$, for increasing values of $\alpha$ or $\lambda$ (i.e. lower degrees of firms' inertia), then the dynamics of the model are characterized by periodic or chaotic oscillations around $E_{*}$. Moreover, under certain circumstances, for instance when BR agents' inertia is less than or equal to LMA agents' inertia, we observed that the action of BR players tends to destabilize the Nash equilibrium. This destabilizing role of the fraction of BR players, can be observed in the bifurcation diagrams of Fig. 1 . where $\lambda=0.9, \alpha=0.7, c=0.1, N=6$ and $r$ (the fraction of BR firms) is taken as bifurcation parameter. Clearly, an increase of the portion of BR players not only destabilizes the Nash equilibrium, thus confirming the conjecture in Bischi et al. 2007, but it also leads to a period-doubling route to chaos. In addition, this numerical exercise shows evidence that for values of $r \approx 0.74$ coexisting attractors are present.
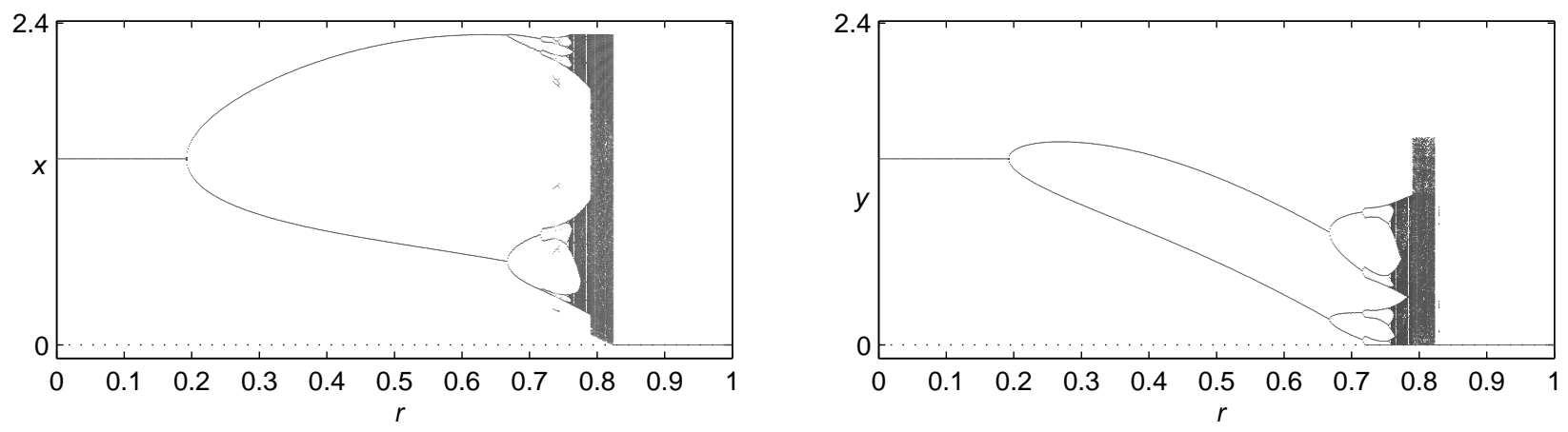

Figure 1: Bifurcation diagrams with parameters $\lambda=0.9, \alpha=0.7, c=0.1, N=6, \epsilon=0$, and $r \in[0,1]$. Left panel shows in the vertical axis the asymptotic values of $x(t)$, the productions of a representative BR firm; Right panel shows the asymptotic values of $y(t)$, the production of a representative LMA firm.

Nevertheless, the BR firms can have a positive effect in terms of global stability of the Nash equilibrium. Indeed, another important indicator for the stability 'robustness' of an attracting set $S$ is the extent of its basin of attraction, denoted by $\mathcal{B}(S)$, which is the set of all initial conditions $(x(0), y(0))$ whose trajectories converge to $S$. With this respect, the presence of BR players tends to increase $\mathcal{B}\left(E_{*}\right)$, the basin of attraction of the Nash equilibrium $E_{*}$, as detailed below.

For showing this property, observe that for the dynamical system (7) with $r=0$ or $r=1$ it is possible to determine analytically the particular rectangular shape of the basin of attraction $\mathcal{B}\left(E_{*}\right)$, which coincides with the feasible region $D$ of the dynamical system itself. In fact, when all firms follow the same behavioral rule, the map (7) assumes a particular form known as triangular (or skew symmetric, or unidirectionally coupled) in the mathematical literature, see e.g. Kolyada, 1992, Dieci et al., 2001 and Dieci et al., 2003. The next proposition clarifies that with sufficiently low inertia, the presence of LMA agents reduces the basin of attraction of the Nash equilibrium $E_{*}$.

Proposition 3. Consider the dynamical system (7) with $N<N_{f}$ [see (9)] so that $E_{*}$ is asymptotically stable. If the following inequality holds

$$
\frac{\lambda^{2}(N-1)}{(\lambda N-1)^{2}}>\frac{2+\alpha(N-1)}{\alpha N^{2}}
$$

then $\mathcal{B}\left(E_{*}\right)$ is always larger in the case of all BR firms than in the case of all LMA firms. 
Proof. See the Appendix.

From this result on global analysis of the model, it follows that, in the particular case in which inertia is neglected, i.e. $\alpha=\lambda=1, \mathcal{B}\left(E_{*}\right)$ is larger in the case of all BR firms than in the case of all LMA firms. More generally, the condition stated in Proposition 3 can be worked out as follows:

- If $\lambda=1 \geq \alpha \geq 0$, then $\mathcal{B}\left(E_{*}\right)$ is larger [smaller] in the case of all BR firms than in the case of all LMA firms provided that

$$
1+\frac{1}{1-2 N}<\alpha \leq 1 \quad\left[0<\alpha<1+\frac{1}{1-2 N}\right]
$$

- If $\alpha=1 \geq \lambda \geq 0$, then $\mathcal{B}\left(E_{*}\right)$ is larger [smaller] in the case of all BR firms than in the case of all LMA firms provided that

$$
\frac{1+N-\sqrt{N^{2}-1}}{2 N}<\lambda \leq 1, \lambda \neq \frac{1}{N} \quad\left[0 \leq \lambda<\frac{1+N-\sqrt{N^{2}-1}}{2 N}\right] .
$$

In other words, if inertia is sufficiently low and Nash equilibrium $E_{*}$ is stable both with all BR agents and with all LMA agents, then the basin of attraction of $E_{*}$ in the case of all BR firms is larger than in the case of all LMA agents. So, there are two contrasting indicators for the ranking of stability of the Nash equilibrium with all firms following the same behavioral rule: an increase of the fraction of BR firms on the one hand can reduce the range of stability of the Nash equilibrium in the parameters space (Proposition 1), but on the other hand can increase the stability of the Nash equilibrium in the state space (Proposition 3).

In order to appraise this kind of stability ranking, let us consider a numerical example such that parameters are given by $\lambda=0.66, \alpha=0.625, c=0.1$ and $N=6$. By Proposition 1 . Nash equilibrium $E_{*}$ is locally stable for any $r \in[0,1]$. In fact, being $\lambda>\alpha, N_{f}$ decreases in $r$, with $N_{f}=7.4$ in case of all LMA firms and $N_{f} \approx 6.06$ in case of all BR firms. This example makes it clear that increasing the fraction of BR players decreases the range of stability of $E_{*}$ in the parameters space. At the same time, by condition (11) in Proposition 3, the presence of only BR players increases (with respect to the case of only LMA players) the range of stability of $E_{*}$ in the state space $(x, y)$ measured by the extent of its basin of attraction $\mathcal{B}\left(E_{*}\right)$, see Fig. 2, where $\mathcal{B}\left(E_{*}\right)$ is depicted in yellow for three different fractions of BR players, namely $r=0$, $r=0.5$ and $r=1$. The yellow region in the right panel (oligopoly with all BR agents) is bigger than the yellow region in the left panel (oligopoly with all LMA agents).
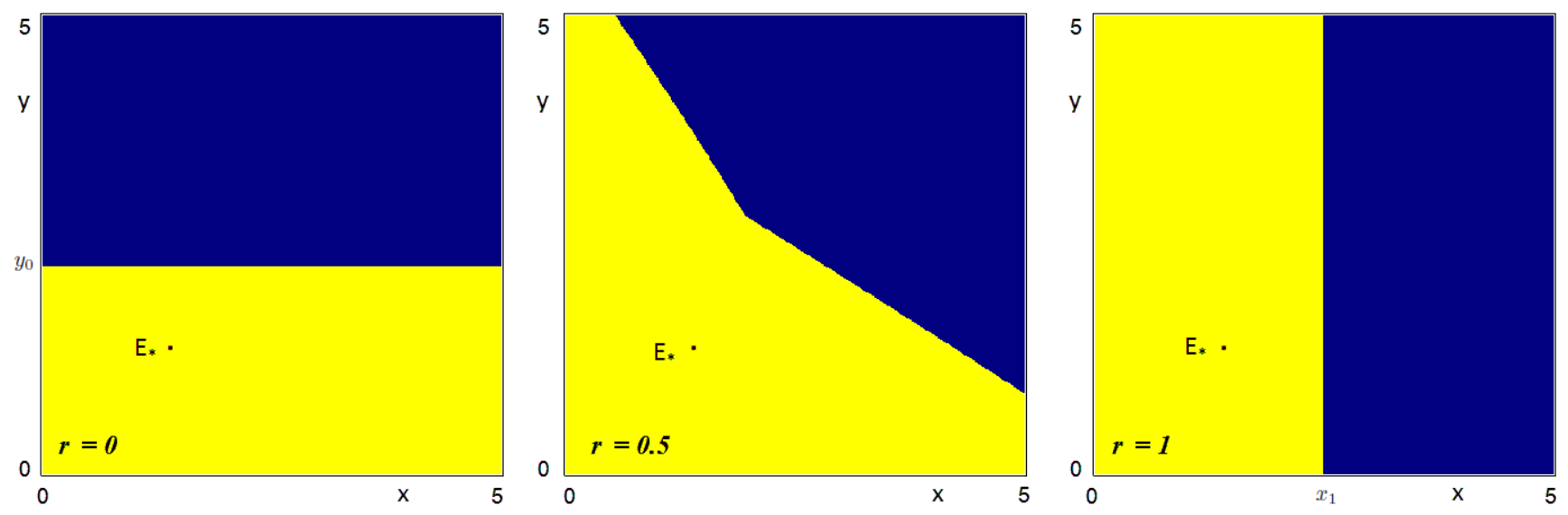

Figure 2: Graphical representation of the basin of attraction $\mathcal{B}\left(E_{*}\right)$ of the Nash equilibrium (yellow region). Parameters are $\lambda=0.66, \alpha=0.625$, $c=0.1, N=6, \epsilon=0$ and $r=0$ (left), $r=0.5$ (center) and $r=1$ (right). Region $\mathbb{R}^{2} / D$ is in blue.

This numerical example underlines that in the oligopoly model 77 the presence of LMA firms may increase the socalled structural stability of the Nash equilibrium, see e.g. Andronov et al., 1967; on one hand, adding more LMA agents reduces the sensitivity of local stability of the Nash equilibrium to variations in the parameters values; on the other hand, it increases the sensitivity of the convergence to the Nash equilibrium to variations on the levels of production. This is a relevant feature of nonlinear models, where local stability does not imply global stability. Hence, to appraise the overall 
effect of the variation of a parameter on the dynamics of the model, it is required to study local stability of attractors as well as their basins of attraction. Exhaustive discussions on this issue for nonlinear oligopoly games can be found, for example, in Huyck et al., 1990, Huyck et al., 1994, Huyck et al., 1997 and Bischi and Kopel, 2001. Concerning the oligopoly model (7), it is characterized by a unique Nash equilibrium and by trajectories leading to unfeasible productions. Thus, the basin of attraction of the Nash equilibrium provides indication on the feasibility of the oligopoly, while the local stability indicates whether the Nash quantity is selected or not. It follows that the two heuristics have to be evaluated on the basis of these two related problems. In particular, LMA firms may have more ability to select the Nash quantity but they may fail to select a positive output, thus making the oligopoly unfeasible for relative large deviations from Nash equilibrium. On the contrary, BR firms may have less ability to select the Nash quantity, but the possibility of an unfeasible oligopoly is reduced, even though production levels do not settle to equilibrium.

It is worth to point out that these considerations and Proposition 3 hold under particular assumptions on the level of inertia and with all firms following the same behavioral rule. Indeed, the degree of inertia and the presence of heterogeneous interacting agents, obtained with $r \in(0,1)$, can lead to much more complicated dynamical properties ${ }^{12}$

Two remarks are in order before concluding this Section. Firstly, from a visual inspection of the bifurcation diagrams in Fig. 1. when the Nash equilibrium $E_{*}$ is unstable, LMA firms produce less than BR firms, i.e. the attractors are located in the region $y<x$. From an economic point of view, this can be explained by noticing that in the presence of a nonlinear and convex demand function such as (2), the linear extrapolation used by LMA firms systematically underestimates the real demand. However, this effect is partly compensated by the monopolistic behavior of LMA firms, as a monopolist tends to produce more than a single oligopolist because it neglects the presence of rivals when setting its production.

Secondly, Proposition 1 states that when the number $N$ of firms is increased the Nash equilibrium loses its stability first when all the firms are BR, and later when all the firms are LMA. Moreover, when all firms are LMA, the attractors that surround the (unstable) Nash equilibrium hit the zero production border for an higher value of $N$ with respect to what happens with all BR firms. This also has a clear economic interpretation, as the LMA firms tend to under-react to production shocks, because they employ a linearization of a convex demand curve. This ensures that LMA production levels do not fluctuate too much far away from the Nash equilibrium as it indeed occurs with many BR firms.

These remarks have relevant consequences in the evolutionary dynamics studied in the next Sections, where the fraction $r=r(t)$ becomes an endogenous dynamic variable and the prevalence of LMA or BR firms over time depends on profits accrued by the two strategies.

\section{The oligopoly with evolutionary competition between behavioral rules}

In this Section, we endogenize the dynamics of the fraction of firms adopting a specific behavioral rule. For doing this, we introduce an evolutionary mechanism that determines how at each time step the fraction $r=r(t)$ changes according to the time average of the differences between past profits accrued to a representative LMA and BR firm:

$$
\Delta \Pi(t)=(1-\omega)\left(\pi_{L M A}(t)-\pi_{B R}(t)\right)+\omega \Delta \Pi(t-1)
$$

where $\omega \in[0,1)$ is a memory parameter that measures the geometric decay rate of past profits, which at time $t$ are given, respectively, by:

$$
\left\{\begin{array}{l}
\pi_{B R}(t)=p(t) x(t)-(c x(t)+K)=\left(\frac{A(t)}{Q(t)}-c\right) x(t)-K \\
\pi_{L M A}(t)=p(t) y(t)-c y(t)=\left(\frac{A(t)}{Q(t)}-c\right) y(t)
\end{array}, \quad A(t) \sim \mathcal{U}[1-\epsilon, 1+\epsilon]\right.
$$

For $\omega=0$ only the current profit gap between the heuristics affects the evolutionary selection process. For $\omega \in(0,1)$ past profits affect 12 with distribution of weights given by $\left((1-\omega),(1-\omega) \omega,(1-\omega) \omega^{2},(1-\omega) \omega^{3}, \ldots\right)$.

Through (12), it follows that more recent profits have stronger impact in the evolutionary selection than older profits. Clearly, an increase of $\omega$ means an increase of memory, in the sense that the influence of past profits on the evolutionary process fades away at a slower rate. We refer the interested reader to Hommes et al., 2012 for further details.

In (13), $Q(t)$ is defined by (3) and $K \geq 0$ represents an information cost for BR agents. As agents need more information and more computational skills for playing BR than LMA, as remarked above, we assume that LMA agents do not bear any

\footnotetext{
${ }^{12}$ Numerical simulations, not reported in this paper for the sake of brevity, show coexisting attractors with complicated topological structure of their basins, thus revealing the possibility of strong path-dependent dynamics of the model.
} 
information and computational cost. This assumption is done without loss of generality, since only the difference of profits enters in the evolutionary equation. Moreover, we assume that $(13)$ are quantities observable by all firms at time $t$.

The evolutionary component of the model describes how $r(t)$, the fraction of BR agents, changes over time according to observed differences of time average profits (12). In particular, we assume that the fraction $r(t)$ is updated according to an exponential replicator equation 13 originally proposed in Cabrales and Sobel, 1992.

$$
r(t+1)=\frac{r(t)}{r(t)+(1-r(t)) e^{\beta \Delta \Pi(t)}}
$$

where parameter $\beta \geq 0$ is referred to as the intensity of choice, which measures how sensitive the players are at selecting profit-increasing behavioral rules. The extreme case $\beta=0$ gives back the model with fixed fractions (7), being $r(t+1)=$ $r(t)=r(0)$. The other extreme case $\beta=\infty$ corresponds to a situation where all firms immediately switch to the behavioral rule showing a (even negligible) better performance, i.e. $r(t) \rightarrow 1$ if $\Delta \Pi(t)<0$ and $r(t) \rightarrow 0$ if $\Delta \Pi(t)>0$.

Combining quantity dynamics (7) with the evolutionary component (14) and the memory equation $(12)$, under the assumption of constant expenditures on the market, namely $\epsilon=0$, a four dimensional discrete-time dynamical system is obtained, which is represented by the iteration of the following map $T: \mathbb{R}^{4} \rightarrow \mathbb{R}^{4}$ :

$$
\left\{\begin{aligned}
x(t+1) & =\max \left\{0,(1-\lambda) x(t)+\lambda\left[\sqrt{\frac{(N-1)[r(t) x(t)+(1-r(t)) y(t)]}{c}}-(N-1)(r(t) x(t)+(1-r(t)) y(t))\right]\right\} \\
y(t+1) & =\max \left\{0,(1-\alpha) y(t)+\frac{\alpha}{2}[y(t)+N(r(t) x(t)+(1-r(t)) y(t))(1-c N(r(t) x(t)+(1-r(t)) y(t)))]\right\} \\
r(t+1) & =\frac{r(t)}{r(t)+(1-r(t)) e^{-\beta \Delta \Pi(t)}} \\
\Delta \Pi(t+1) & =(1-\omega)\left[\pi_{B R}(t+1)-\pi_{L M A}(t+1)\right]+\omega \Delta \Pi(t)
\end{aligned}\right.
$$

Model (15) describes the dynamics of the evolutionary oligopoly with heterogeneous firms endogenously selecting between BR and LMA heuristics. In the following, we study the stability properties of this model and show, by analytical and numerical methods, the consequent choices of firms in terms of heuristic to adopt and quantities to deliver in the market.

It is worth to point out that for the special case $\omega=0$ (no memory), $\Delta \Pi(t)$ depends only on $\pi_{B R}(t)$ and $\pi_{L M A}(t)$, which are functions of $x(t), y(t)$ and $r(t)$, but not on $\Delta \Pi(t-1)$. It follows that map $T$ in 15 reduces to a three dimensional dynamical system that evolves inside the three-dimensional phase space $(x, y, r) \in \mathbb{R}_{+}^{2} \times[0,1]$, where $\mathbb{R}_{+}=[0,+\infty)$. The boundary planes $r=0$ and $r=1$, where only one pure behavioral strategy is employed (LMA or BR respectively), are invariant sets of the map. On these planes, the dynamics are governed by the two-dimensional restrictions of (15) on them, given by the triangular maps (19) and (21) respectively. Observe that an attractor on one of these two-dimensional restrictions of the phase space may be transversely stable, so that it attracts trajectories starting outside the restriction, i.e. from $r(0) \in(0,1)$; in this case, the attractor on the restriction is also an attractor of map $T$ in 15$)$. Otherwise, the attractor on the restriction is transversely unstable, so that it may not be reached by trajectories coming from inside the phase space, but only with initial conditions such that $r(0)=0$ or $r(0)=1$. From 15 with $\omega=0$, it is straightforward to see that the steady states of the evolutionary model are obtained for $r=0, r=1$ and any $r^{*} \in(0,1)$ for which $\pi_{B R}=\pi_{L M A}$. The following proposition studies existence and local stability of the Nash equilibria of this three-dimensional evolutionary model when memory is neglected.

Proposition 4. Consider the dynamical system (15) with $\omega=0$ (i.e. "without memory"). Map (15) becomes a threedimensional dynamical system such that:

- If $K=0$, then a continuum of equilibrium points $E_{*}$ fills the segment $E=\left\{x=\frac{N-1}{c N^{2}}, y=\frac{N-1}{c N^{2}}, 0 \leq r \leq 1\right\}$. These equilibria are stable as long as the stability conditions for the Nash equilibrium stated in Proposition 1 are satisfied for all $r \in[0,1] 14$

\footnotetext{
${ }^{13}$ The exponential function is also a simple and useful way to avoid negative values of fitness, see e.g. Hofbauer and Weibull 1996 and Hofbauer and Sigmund, 2003 Recently, in Kopel et al. 2014 the same specification of 14 has been considered in an evolutionary oligopoly model with firms choosing between a standard and a social responsible behavior.

${ }^{14}$ By stable equilibrium we mean "Lyapunov stable" or "non-asymptotic stable", see e.g. Kuznetsov 1998 Roughly speaking, any trajectory starting in a neighborhood of $E$ does not necessary reach $E$ but it remains closed to it.
} 
- If $K>0$, then only the two extreme points of the segment $E$ are equilibria, namely

$$
E_{0}=\left(\frac{N-1}{c N^{2}}, \frac{N-1}{c N^{2}}, 0\right) \text { and } E_{1}=\left(\frac{N-1}{c N^{2}}, \frac{N-1}{c N^{2}}, 1\right)
$$

in which all agents adopt the same strategy, which is LMA or BR respectively. Provided that the conditions of Proposition 1 for the stability of the Nash equilibrium $E_{*}$ are satisfied for $r=0$, equilibrium $E_{0}$ is asymptotically stable, whereas $E_{1}$ is unstable.

Proof. See the Appendix.

From Proposition 4, we notice that, with positive information cost $K>0$, LMA dominates BR whenever the productions are at the Nash equilibrium, since, in that case, it is $\pi_{B R}<\pi_{L M A}$. Thus, due to the presence of an information cost for playing $\mathrm{BR}$, equilibrium $E_{1}$ cannot be stable. In other words, starting from a game with $r=1$, i.e. with all agents playing $\mathrm{BR}$, any small "mutation" leading to a few agents playing LMA will grow up under the evolutionary pressure induced by (14), since BR is dominated by LMA because of information costs.

In the presence of memory, i.e. with $\omega \in(0,1)$, the dynamics of model 15$)$ evolve in the four-dimensional phase space $(x, y, r, \Delta \Pi) \in \mathbb{R}_{+}^{2} \times[0,1] \times \mathbb{R}$. Nevertheless, its dynamical features are similar to the case $\omega=0$. Indeed, even with $\omega \in(0,1)$ the subspaces $r=0$ and $r=1$ are two invariant regions where only one pure behavioral strategy is employed and where dynamics are governed by triangular maps (19) and (21) respectively. Moreover, analogous stability conditions of Nash equilibria hold, as stated in the following proposition.

Proposition 5. Consider the dynamical system (15) with $\omega \in(0,1)$ (i.e. "with memory").

- If $K=0$, then a continuum of equilibrium points $E_{*}$ fills the segment $E=\left\{x=\frac{N-1}{c N^{2}}, y=\frac{N-1}{c N^{2}}, 0 \leq r \leq 1, \Delta \Pi=0\right\}$.

- If $K>0$, then only the two extreme points of the segment $E$ are equilibria, namely ${ }^{15}$

$$
E_{0}=\left(\frac{N-1}{c N^{2}}, \frac{N-1}{c N^{2}}, 0, K\right) \text { and } E_{1}=\left(\frac{N-1}{c N^{2}}, \frac{N-1}{c N^{2}}, 1, K\right)
$$

in which all agents adopt the same strategy, which is LMA or BR respectively.

Stability conditions for Nash equilibria $E_{*}, E_{0}$ and $E_{1}$ are as in Proposition 母.

Proof. See the Appendix.

It is worth to point out that Propositions 4 and 5 state that for $N>N_{f}$ the Nash equilibrium cannot be stable, where $N_{f}$ is given in (9) as a function of $\alpha, \lambda$ and $r$, confirming the results in Hommes et al., 2011 on the destabilizing role of the number of players $N$ in evolutionary oligopoly games, see also Droste et al. [2002. However, the concern here is not the effect of the number of players but the dependence of $N_{f}$ on $r$ and the effects of the evolutionary competition between the two heuristics and the related economic implications. The topic is examined in depth in the following Section.

\section{Dynamics of the evolutionary oligopoly model}

Let us begin our discussion on the model when no information costs are present. In this case, there are time evolutions of the system that lead to asymptotic coexistence of both BR and LMA behavioral rules. In other words, even if there is no extra cost for playing the "more knowledge-demanding" BR strategy, a finite fraction of firms could find more useful to continue adopting the LMA rule of thumb. This result may sound quite strange at a first sight, but the intuition behind it lies in the fact that both strategies are boundedly rational. Thus, in certain cases the more sophisticated BR strategy may be counterproductive and discarded in favor of the LMA strategy, although the latter requires less information, employs a linear approximation of the demand function and completely ignores the presence of competitors at each step. Another

\footnotetext{
${ }^{15}$ In Cournot oligopoly games, a Nash equilibrium is identified by firms' productions only. With a slight abuse of terminology, we refer to equilibria $E_{*}, E_{0}$ and $E_{1}$ as Nash equilibria. Moreover, as no confusion arises, we use the same notation to indicate the equilibria of the model 7), the equilibria of model 15 with $\omega=0$ and the equilibria of the model 15 with $\omega \in(0,1)$. Note that model 7 can be seen as a particular case of model 15 with $\beta=\omega=0$.
} 
interesting feature when information costs are neglected is that the asymptotic values of $r(t)$ are strongly influenced by the initial conditions of the system; in other words, path dependence occurs. This is related to the statements given in Proposition 4 (model without memory) and Proposition 5 (model with memory) that with $K=0$ a segment of stable equilibria exists. From an economic point of view, in this segment of equilibria an isoprofit condition holds, so that the role of transient is crucial: any initial perturbation modifies the whole trajectory that follows, thus leading to a different long-run value of $r(t)$. This effect has been numerically confirmed by the observed asymptotic fate of several trajectories obtained with different initial conditions, as shown in Fig. 3, where two bifurcation diagrams are depicted to show the asymptotic values of $r$ for increasing values of $N$; different colors correspond to different initial conditions, both with different values of $r(0)$ (left panel) and with different values of $(x(0), y(0))$ (right panel). In all numerical examples, we fixed $\Delta \Pi(0)=0$ as any other choice would be too much arbitrary.

On the basis of this argument, we may expect that the LMA rule becomes a weaker strategy as $N$ increases, because it is less and less accurate as each LMA player neglects the presence of more and more competitors when $N$ is higher. Indeed, the situation is not so simple, as the following numerical simulations show. What is worth of note is that when the Nash equilibrium loses stability for increasing values of $N$, the prevailing behavior is characterized by $r=1$, i.e. all firms play the BR strategy. In other words, in the absence of information costs and outside the Nash equilibrium, the (pure) BR strategy dominates the LMA strategy. This is consistent with the remark given at the end of the previous Section, where we noticed that the non-equilibrium dynamics observed when the Nash equilibrium is unstable are characterized by $y(t)<x(t)$. In fact, LMA firms systematically underestimate the demand, due to their linear extrapolation of the convex demand, and lower production of LMA translates into lower profits, having all agents the same production and information costs and the same selling price.

A comparison of trajectories for the model with memory and without memory suggests that they almost overlap and are almost indistinguishable, see Fig. 3 . The only difference that can be captured is a slightly higher propensity to employ the BR heuristic in the evolutionary selection process when memory of past profits is introduced. This minimal difference is due to the fact that BR firms perform better (at least on average) along the trajectory leading to the Nash equilibrium.
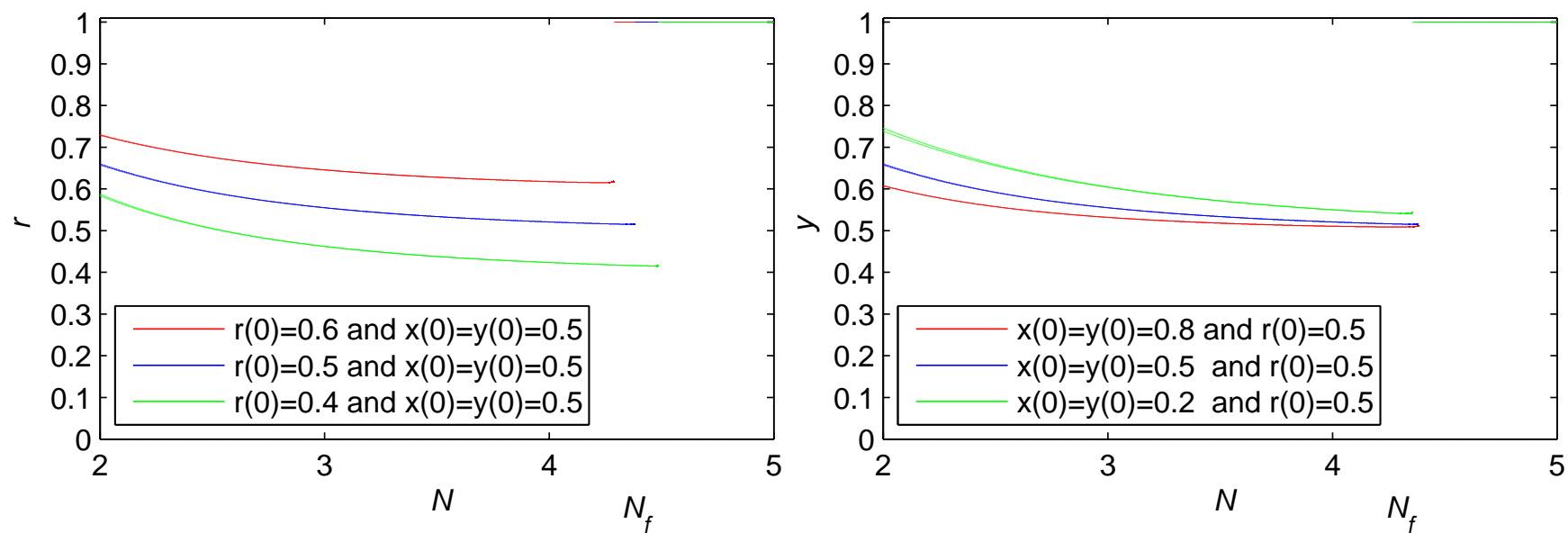

Figure 3: Bifurcation diagram for increasing values of $N$ showing the asymptotic values of $r$. Different colors correspond to different initial conditions, both with different values of $r(0)$ (left panel) and with different values of $(x(0), y(0)$ ) (right panel). Parameters are $\lambda=\alpha=1$, $c=0.1, \beta=1, K=0, \epsilon=0$. Both the cases $\omega=0$ and $\omega=0.5$ are considered. The differences between those two cases are negligible and the trajectories are almost indistinguishable (overlapped), although with memory the final value of $r$ is slightly closer to 1 .

Introducing the information costs for playing BR, the more interesting, and even intriguing, dynamic scenarios are obtained when the Nash equilibrium loses stability and oscillatory (periodic or chaotic) asymptotic motions occur along attractors, which are located on the two-dimensional invariant planes $r=0$ or $r=1$, as well as in the interior regions of the phase space, where $r$ is intermediate. To perform some numerical explorations, we choose a set of parameters that is representative of the most interesting phenomena we detected in the evolutionary model. We start with BR firms having a lower speed of adjustment than LMA firms, i.e. $\lambda<\alpha$ with $\lambda=0.6, \alpha=0.7, c=0.1, \beta=1, K=0.01$ and $\omega=0$. The 
corresponding bifurcation diagrams are depicted in Fig. 4a, where the number of firms $N$ is the bifurcation parameter and in the vertical axes the asymptotic productions by BR firms $x$, LMA firms $y$, and fractions $r$ of BR firms are respectively shown. Because of the information cost for playing BR, as long as the Nash equilibrium is stable, i.e. when $N<N_{f}$, the long run value of $r(t)$ converges to 0 . The economic explanation of this outcome is that at the Nash equilibrium both BR and LMA firms produce the same quantity and, due to the setting of the oligopoly, the only difference in terms of profits is the extra cost $K$ payed by BR firms to be more informed. It follows that once all the firms produce the Nash quantity, it is convenient not to pay for more information and become LMA. In other words, at the Nash equilibrium BR behavior is dominated by LMA one. Instead, when the Nash equilibrium $E_{0}$ loses stability through the period-doubling bifurcation, the attractor of the system is a cycle of period two, which is transversely stable for small additional increments of $N$ and loses its transverse stability when $N$ is further increased. As a consequence, $r(t)$ moves towards the other invariant plane $r=1$, where a different cycle of period two exists, which is located around $E_{1}$. This is observable in the 'jump' in the bifurcation diagram of Fig. 4a Then, for higher values of $N$, the usual period-doubling cascade to chaos occurs, with a chaotic attractor entirely located on the invariant plane $r=1$, where all firms choose to behave according to the BR rule. The numerical example underlines that the BR behavior dominates the LMA one when the oligopoly dynamics are far away from the Nash equilibrium. In fact, when the oligopoly is profitable, namely $p(t)-c>0$, the heuristic that leads to produce more quantities, in this specific case the BR heuristic, is also the more profitable. It is worth to observe that, in this example, for $N \geq 9$ the oligopoly is unfeasible, which means that all firms prefer to stop production.

This numerical example underlines one of the most interesting feature of the evolutionary competition when information costs are present: firms select the LMA heuristic when convergence to the Nash equilibrium occurs, whereas they select the BR heuristic when the system fails to converge to the Nash equilibrium. Despite this propensity to switch from LMA to BR when the Nash equilibrium becomes unstable, the transition is not always driven by sudden jumps in the fraction $r(t)$, but depends on the speeds of adjustment and on the amount of information costs. In particular, a lower speed of adjustment of BR firms, which implies their higher propensity to stuck to previous production decisions, reduces the positive production gap between BR and LMA firms. This effect, added to an increased information cost, reduces the profitability of the BR heuristic, with a consequent reduction of firms selecting it. In particular, even with positive information costs $K>0$, it is possible to exhibit examples with intermediate asymptotic values of $r$ as confirmed by numerical simulations. This implies the existence and the asymptotic stability of non-equilibrium attractors (periodic or chaotic) located in the interior of the phase space, in which a positive fraction of firms always employs each heuristic. As an example, let us consider the bifurcation diagram in Fig. 4b obtained for parameters as in Fig. 4a but with a lower speed of adjustment of BR firms, namely $\lambda=0.5$ instead of $\lambda=0.6$. As it can be seen, the trajectories in the bifurcation diagrams indicate that, for $N>9$, intermediate values of the fraction $r(t)$ are obtained in the long run, suggesting an asymptotic coexistence of BR and LMA players. By comparing the levels of production for BR firms, $x(t)$, and LMA firms, $y(t)$, for the two different levels of the speed of adjustment $\lambda$ (see the first and second row in Fig. 4), we observe that differences in production between the two heuristics are not so marked for a low speed of adjustment. It follows that the extra profits coming by exploiting more information dwindle in disequilibrium dynamics and the extra costs of information prevent the evolutionary dominance of BR over LMA. For $N \leq 11$ at least $50 \%$ of the firms adopt the LMA heuristic, while for $N \geq 12$, the oligopoly becomes unfeasible. It follows that for a low speed of adjustment $\lambda$ and a sufficiently high information cost $K$, the BR heuristic is never dominant. Another interesting aspect regards the feasibility of the oligopoly under the two heuristics. As observable from a comparison of the bifurcation diagrams of the right and left columns of Fig. 4, the LMA heuristic allows the feasibility of the oligopoly with a larger number of firms compared to the BR heuristic. Indeed, when the LMA heuristic is more desirable from an evolutionary point of view, as it is in the bifurcation diagrams of the second column of Fig. 4. the feasibility of the oligopoly is lost for $N>11$, while when the LMA heuristic is less desirable from an evolutionary point of view, as it is in the bifurcation diagrams of the first column of the same Figure, the feasibility of the oligopoly is lost already for $N>8$. This is imputable, in the proposed numerical examples, to the lower reactivity of the LMA heuristic in term of productions. Identical results and considerations are obtained with memory, namely $\omega \in(0,1)$. Therefore and for the sake of brevity, the related numerical examples are omitted.

In addition to the discussed properties of the evolutionary oligopoly, there is at least another important characteristic of the dynamics of the model arising when both Nash equilibria $E_{0}$ and $E_{1}$ are unstable. Indeed, for a given range of $N$, attractors of the system coexist, each located on one of the two invariant planes respectively and each with its own basin of attraction. This means that, for a given set of parameters, starting from two different initial conditions with intermediate values $r(0) \in(0,1)$, the corresponding trajectories may converge to different coexisting attractors where opposite behavioral rules prevail.

This is the situation shown in Fig. 5a, where the parameters are as in the bifurcation diagram of Fig. $4 \mathrm{a}$ and $N=8$. The numerical example shows the sensitivity to initial conditions of productions in the evolutionary oligopoly. The two 
depicted trajectories start from two different initial conditions with same $x(0)$ and $y(0)$ but different initial distribution of heuristics $r(0)$. The trajectory starting from $r(0)=0.01$ converges to a cycle of period 2 located on the invariant plane $r=0$, whereas the trajectory starting from $r(0)=0.2$ converges to a chaotic attractor located on the invariant plane $r=1$. A similar situation occurs also assuming the same speed of adjustment for the two heuristics, i.e. $\alpha=\lambda$. For example, for parameters as in Fig. 5a but $\lambda=\alpha=0.3$ and $N=15$, two different initial conditions (represented by the empty dots) lead the system to two different coexisting stable cycles of period 2, which are located on the invariant planes $r=0$ and $r=1$. Both attractors are represented by black points in the figure. The motion on the two invariant planes are governed by the two triangular maps (19) and (21) respectively, so their properties can be easily studied (see e.g. Kolyada, 1992 and Dieci et al. 2001).

These numerical simulations highlight the importance of history on the selection of heuristics and underline that evolutionary selection of one particular heuristic is a path-dependent process as well as the long-run outcome of the industry.
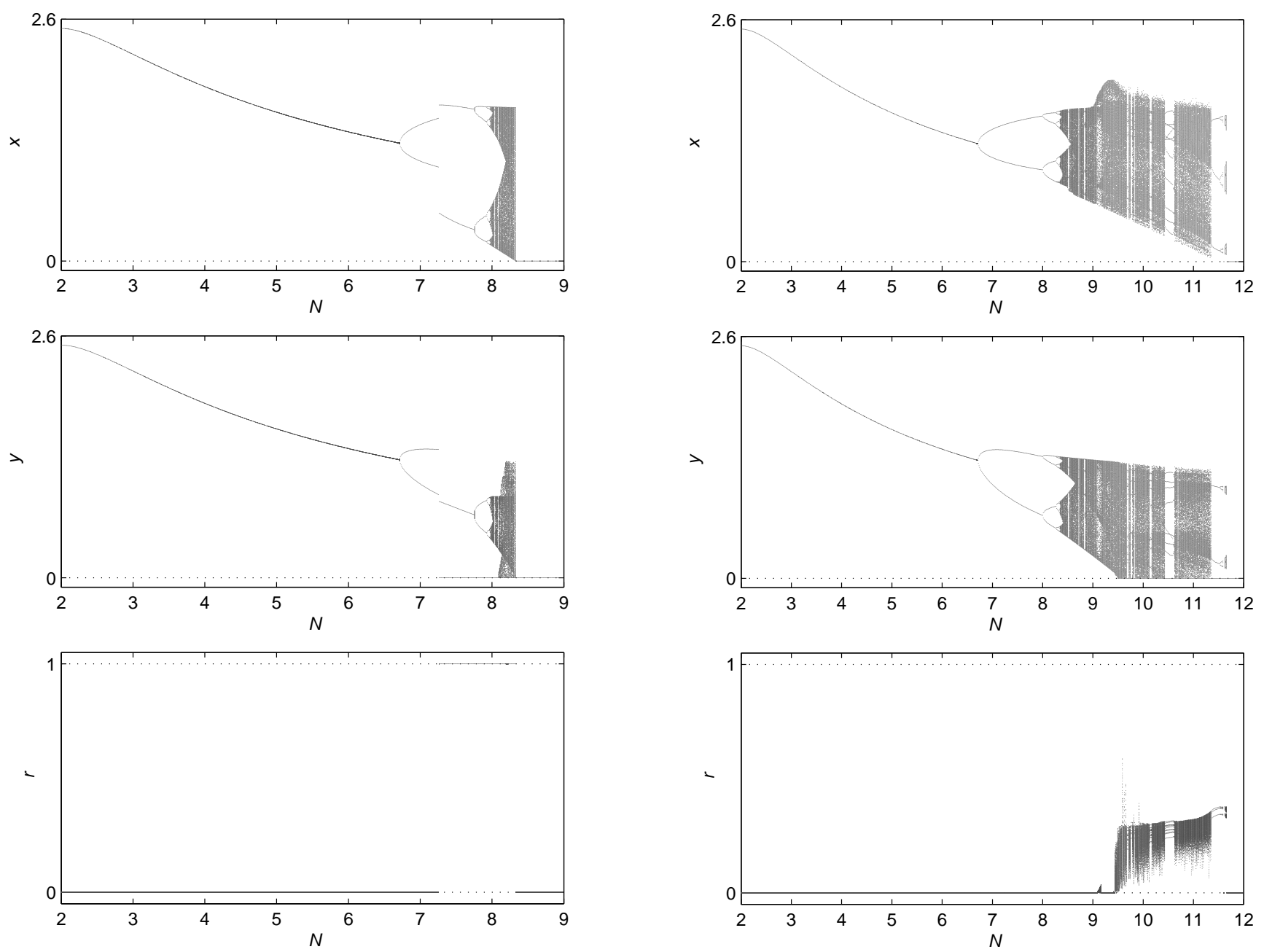

(a)

(b)

Figure 4: Bifurcation diagrams such that 3000 asymptotic values (after a transient of 2000 iterations) are represented for the production of BR firms $x$ (first row), for the production of the LMA firms $y$ (second row) and for the fraction $r$ of BR firms (third row). 4a Parameters $\lambda=0.6$, $\alpha=0.7, c=0.1, \beta=1, \epsilon=0$, and $K=0.01$ and bifurcation parameter $N \in[2,9]$. For increasing $N$, the jump corresponds to the lost of transverse stability of the 2-cycle located in the invariant plane $r=0$. $4 \mathrm{~b}$ Parameters $\lambda=0.5, \alpha=0.7, c=0.1, \beta=1, \epsilon=0, K=0.1$ and bifurcation parameter $N \in[2,12]$. For $N>9$, intermediate values of the fraction $r(t)$ are obtained in the long run, with coexistence of BR and LMA players. The numerical examples regard model 15 with $\omega=0$ (no memory) and initial condition $(x(0), y(0), r(0))=(0.1,0.2,0.5)$. 
Indeed, the heuristic selected by firms influences the level of production outside the Nash equilibrium, the level of profits and, most notably, the feasibility of the oligopoly itself, as previously observed and discussed. In addition, the proposed numerical examples show that the evolutionary dominance of one heuristic over the other may also depend on the initial diffusion of heuristics among the firms populating the oligopoly.

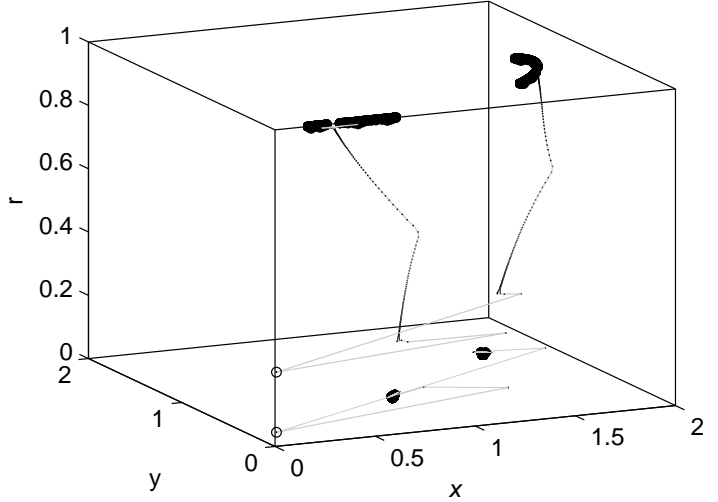

(a)

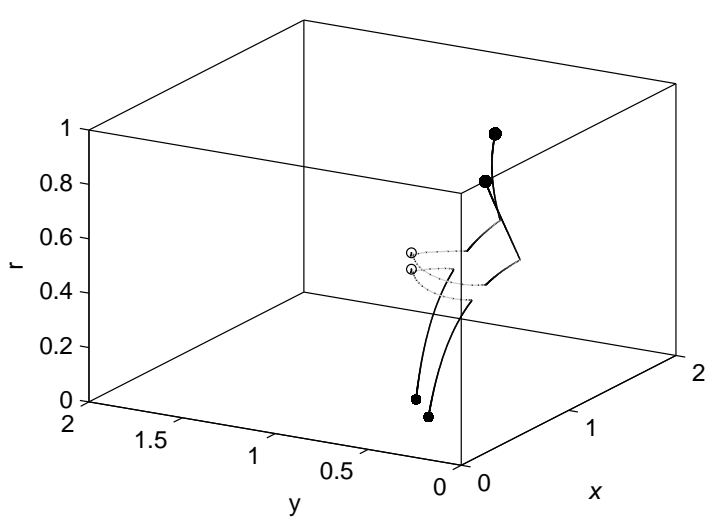

(b)

Figure 5: Trajectories on the three-dimensional phase space of evolutionary oligopoly 15 without memory $(\omega=0)$. 5a: two different initial conditions, $(0.1,0.2,0.2)$ and $(0.1,0.2,0.01)$ (empty dots), lead to two different coexisting attractors: a 2-cycle and a chaotic attractors respectively on the invariant planes $r=0$ and $r=1$; parameters are as in Fig. 4a with $N=8$. (5b): two different initial conditions, $\left(\frac{N-1}{c N^{2}}+0.01, \frac{N-1}{c N^{2}}+0.01,0.58\right)$ and $\left(\frac{N-1}{c N^{2}}+0.01, \frac{N-1}{c N^{2}}+0.01,0.52\right)$ (empty dots), lead to two different coexisting stable cycles of period 2 , located on the invariant planes $r=0$ and $r=1$; parameters are $\lambda=\alpha=0.3, c=0.1, \beta=1, K=0.01, N=15$.

To analyze in which measure the 'historical accidents' can influence the evolutionary process of heuristic selection, we study the basins of the two attractors located on the invariant planes. These are three-dimensional sets that can be represented in a plane by sections with constant $r$. For example, with the same set of parameters used in Fig. 5b, the first row of Fig. 6 depicts four different sections of the basins. The points in the red region generate trajectories converging to the cycle of period 2 located on the invariant plane $r=0$, the green region is the basin of the 2-cycle on the plane $r=1$ and the blue region, as before, represents the points whose trajectories converge to levels with zero production. It is worth to observe that the sections of the basins on the invariant planes $r=0$ and $r=1$ can be easily computed according to (20) and (22) respectively ${ }^{16}$ Moreover, the portions of the phase space with values of $r$ sufficiently close to an invariant surface generate trajectories that converge to the closer pure behavioral strategy. In other words, if a trajectory starts with prevalence of BR players, then in the long run the oligopoly market will converge to a situation with all BR players, and vice-versa for LMA players. However, more uncertain cases occur for intermediate values of $r(0)$, as in such cases also the initial production levels $(x(0), y(0))$ influence the asymptotic fate of the fraction $r(t)$. Of course, by properly tuning the information cost parameter $K$, the dominance of behavioral rules can be changed. In this case, it is easy to obtain examples where all trajectories with initial conditions $r(0) \in(0,1)$ converge to an attractor on one of the two invariant planes. The investigation of the basins of attraction through the technique of cut sections shown in Fig. 6] underlines a strong sensitivity on initial conditions of the evolutionary selection and of the long-run outcome of the industry. It follows that the selection of one particular heuristic over the other may depend more on 'historical accidents' than on a real superiority of the heuristic itself. As stated in Proposition 5 the existence and stability of Nash equilibria do not depend on the presence of memory in the evolutionary process. Moreover, omitted numerical simulations show that the attractors are not essentially influenced by the memory even in situation of disequilibrium. However, the presence of memory can influence the long-run outcome of the dynamic system. This feature in the evolutionary game is clearly observable in the basins of attraction depicted in Fig. 6. From a comparisons of the sections of the basins of attraction of the first row, obtained without memory, and the ones of the second row, obtained with memory, we observe that when agents take into account time averages of past profits as a measure to select different heuristics, the possibility that all the firms adopt the BR is reduced whereas it is more likely that all the firms become LMA in the long run.

\footnotetext{
${ }^{16}$ Note that condition 11 of Proposition 3 continues to hold in the present example, so that the red regions in the leftmost panels of Fig. 6 are larger than the green regions in the rightmost panels of the same Figure.
} 


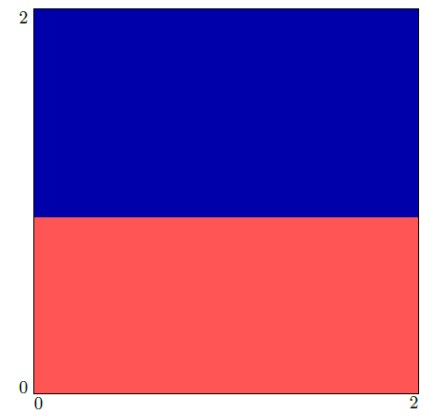

(a) $r=0$

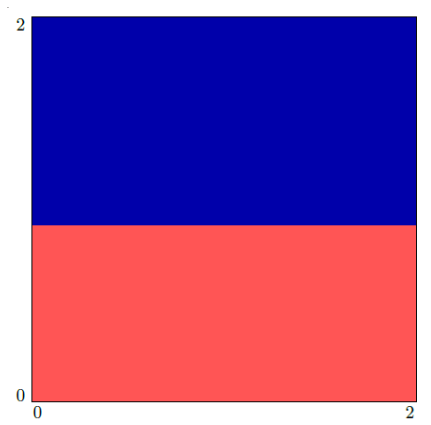

(e) $r=0$

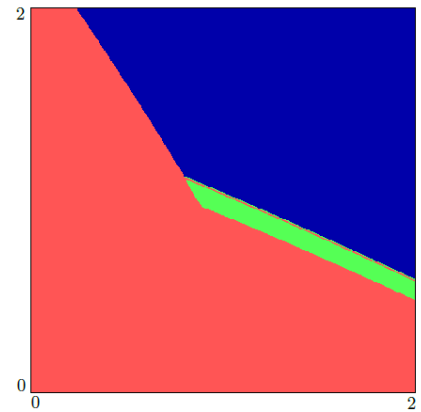

(b) $\mathrm{r}=0.5$

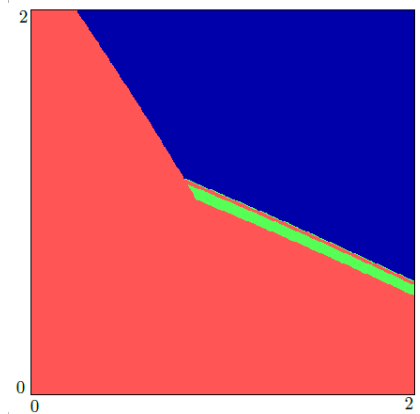

(f) $\mathrm{r}=0.5$

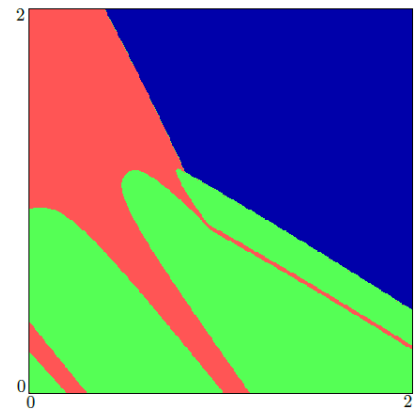

(c) $\mathrm{r}=0.55$

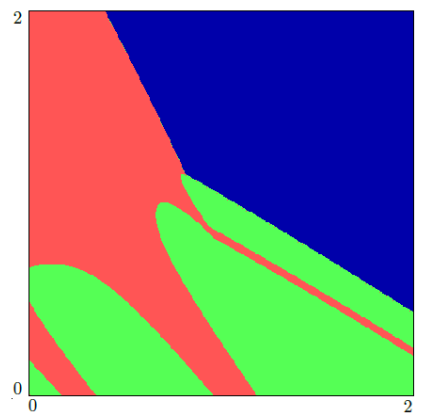

(g) $\mathrm{r}=0.55$

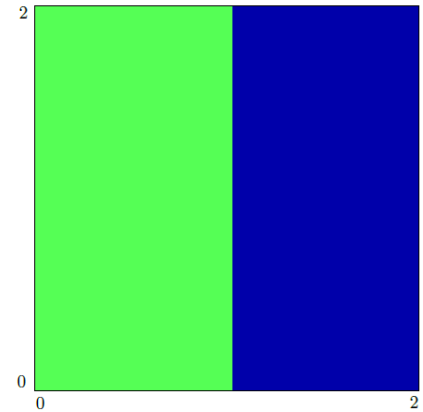

(d) $r=1$

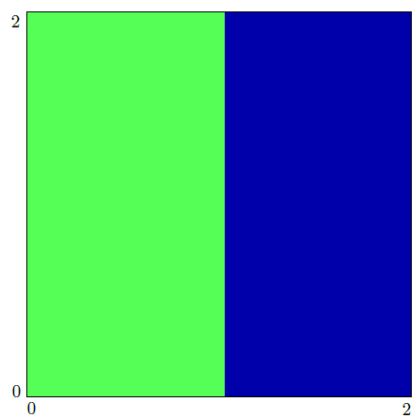

(h) $r=1$

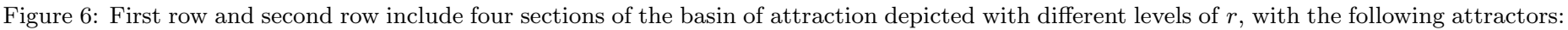

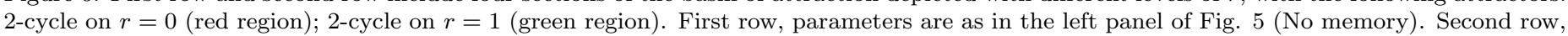

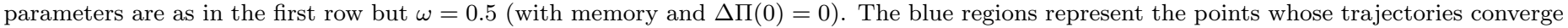
to $(0,0)$.

In order to have an illustration of the oligopoly dynamics for the main economic variables along the motion that prevails in the long run, we show the time series generated by the system, i.e. the versus-time sequences of $x(t), y(t)$ and $r(t)$ computed along a typical trajectory, together with $x(t)-y(t)$ and $\pi_{B R}(t)-\pi_{L M A}(t)$. We focus on the case of an inner stable attractor to clarify the reasons of evolutionary heterogeneity. In particular, in Fig. 7a the same set of parameters of the bifurcation diagrams of Fig. $4 \mathrm{~b}$ with $N=10$ is employed. As it can be seen, the asymptotic dynamics of $x(t)$ and $y(t)$ are chaotic (around the Nash equilibrium) with oscillations of large amplitude, and the time evolution of the fraction $r(t)$ exhibits bounded oscillations eventually in the range $(0.15,0.30)$ : the evolutionary pressure based on observed profits gives rise to behavioral heterogeneity in firms' choices, with a mixed fraction between $15 \%$ and $30 \%$ of BR players.

The heterogeneity in firms' choices admits a clear economic explanation. From Fig. 7a we observe that BR firms produce always more than LMA firms. It follows that as long as $p(t)>c$, both BR firms and LMA firms gain positive profits, with the BR heuristic leading to higher profits, namely $\pi_{B R}>\pi_{L M A}$. On the contrary, when $p(t)<c$, both BR firms and LMA firms suffer losses, with the LMA heuristic being less "harmful" than the BR heuristic, namely $\pi_{B R}<\pi_{L M A}$. It follows that to have evolutionary heterogeneity it is required that the oligopoly alternates periods of profitability with periods of losses in such a way that extra earnings made by BR firms in periods of positive profits are compensated by more limited losses by LMA firms in periods of negative profits. This profit dynamics is clearly observable in the last line of Fig. 7a.

The times series analysis underlines an important feature of evolutionary competition between BR and LMA heuristics in the evolutionary oligopoly (15): The LMA heuristics, as long as it leads to produce less than the BR heuristic, can spread through the population only when the oligopoly is not profitable. This mechanism resembles the "spiteful behavior" detected in evolutionary oligopolies by, for example, Vega-Redondo, 1997, Vriend, 2000, and Vallée and Yildizoglu, 2009, to explain evolutionary dominance of Walrasian behavior over Cournotian behavior. Roughly speaking, the spiteful behavior is such that a strategy is evolutionary stable because it allows to reduce losses respect to other strategies. The same can occur in the evolutionary oligopoly 15 for the LMA heuristic. In particular for this oligopoly setting, we can summarize the result as follows:

Proposition 6. Consider the evolutionary oligopoly with isoelastic inverse demand (2) and cost function (1) with $K_{i}=0$. 

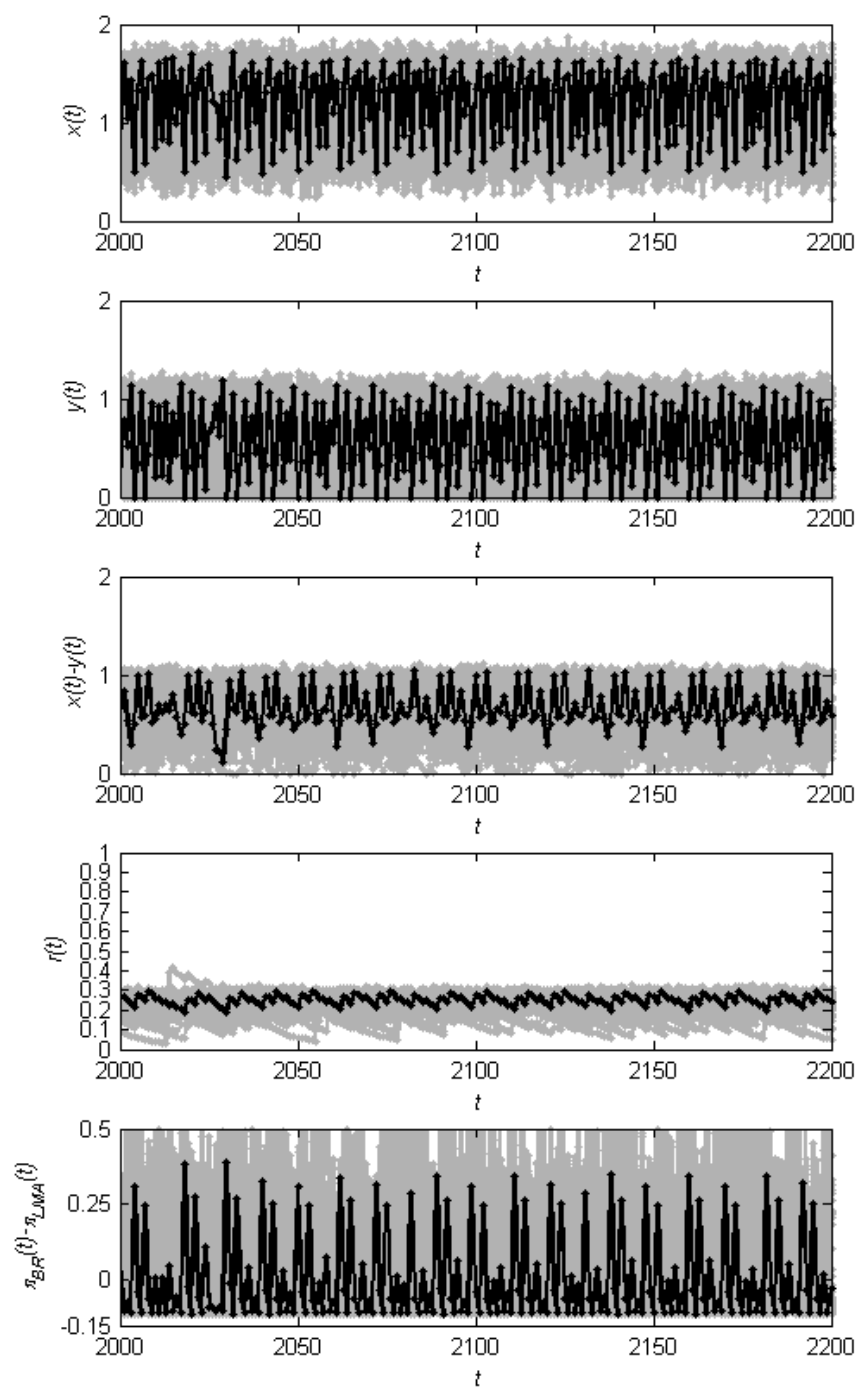

(a) $\omega=0$
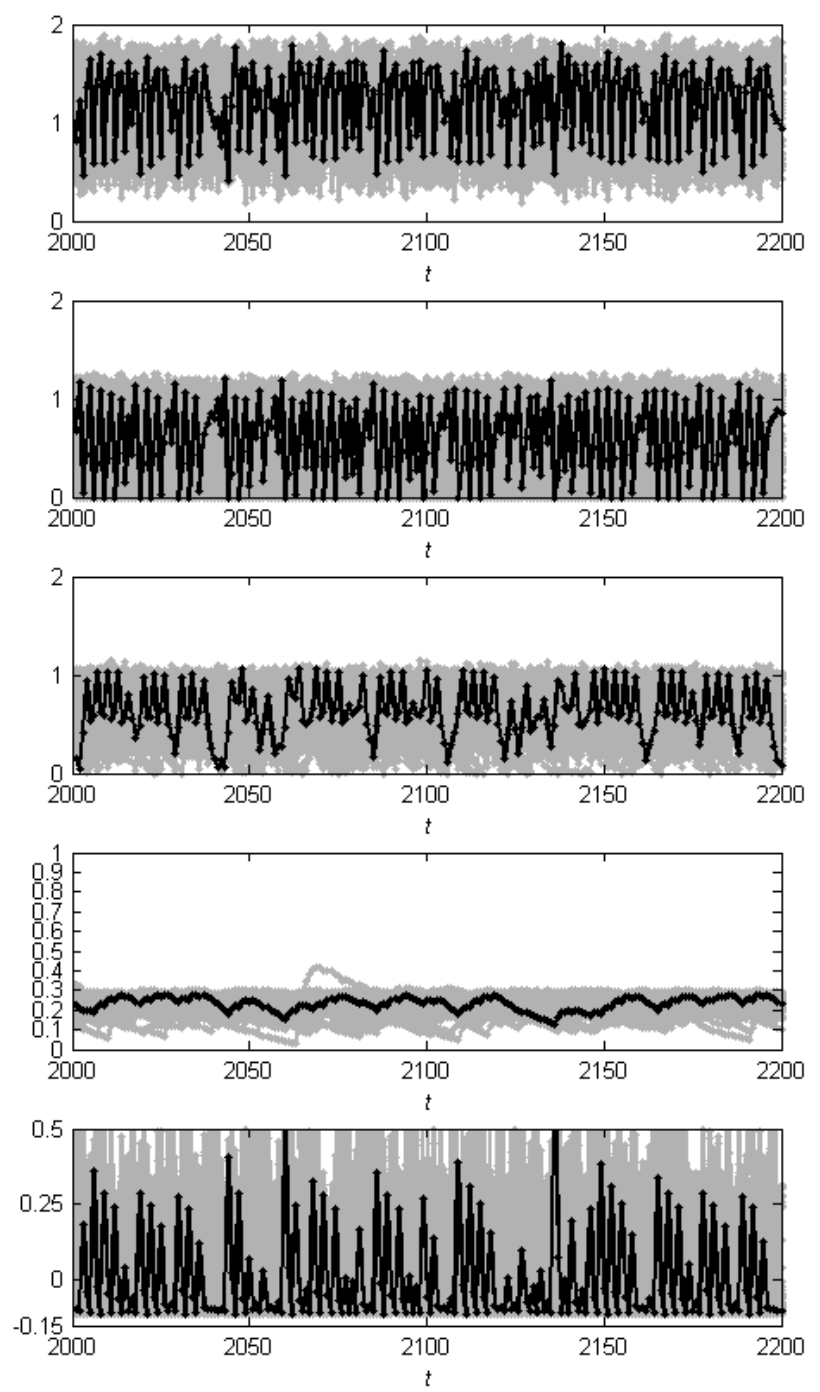

(b) $\omega=0.5$

Figure 7: Versus-time sequences of $x(t), y(t), x(t)-y(t), r(t)$ and $\pi_{B R}(t)-\pi_{L M A}(t)$ along a typical trajectory after a transient of 2000 iterations. In black $\epsilon=0$. In gray 100 runs for $A(t) \sim \mathcal{U}(1-\epsilon, 1+\epsilon)$, with $\epsilon=0.05$. 7ap: Parameters as in Fig. 4b with $N=10$. $7 \mathrm{~b}$ : Parameters as in Fig. 7 but with memory $\omega=0.5$.

Then, the heuristic that leads to produce more (the more aggressive strategy) is evolutionary dominant if the oligopoly is profitable, i.e. $p(t)-c>0$. On the contrary, the heuristic that leads to produce less (the less aggressive strategy) can spread among the population if the oligopoly is not profitable, i.e. $p(t)-c<0$.

The proof of the proposition is trivial observing that, in such an oligopoly, firms adopting different heuristics earn the same returns for unit of production.

The memory does not wash out firms' heterogeneity of choices, as highlighted by the times series of Fig. 7b obtained with $\omega=0.5$ and the other parameters as in Fig. 7a. Moreover, the heterogeneity is observable also when the evolutionary oligopoly is forced by noise. In particular, assuming randomness in the aggregate consumers' expenditures, namely $1>\epsilon>0$, and combining quantity dynamics (7) with the evolutionary component (14) and the memory equation $(12)$, a system of equations is obtained that induces a four-dimensional dynamical process in $\{x(t), y(t), r(t), \Delta \Pi(t)\}(t)$ with noise parameter 
$A(t) \sim \mathcal{U}(1-\epsilon, 1+\epsilon){ }^{17}$ This system is clearly a stochastic version of the evolutionary oligopoly model $(15)$. Monte Carlo simulations show the persistence of evolutionary heterogeneity in each realization, see gray paths in Fig. 7a (without memory) and gray paths in Fig. $7 \mathrm{~b}$ (with memory) 18

In Fig. 8 the same parameters are considered but the information cost is increased to the value $K=0.8$. The motion of quantities $x(t)$ and $y(t)$ is again chaotic with oscillations of amplitude larger than before. However, it is the time evolution of $r(t)$ to be quite counterintuitive, as it exhibits large oscillations between values very close to 0 and values very close to 1, with a typical phenomenon of on-off intermittency (see e.g. Ashwin et al., 1996, Venkataramani et al., 1996 and Bischi et al. 1998) before finally settling on the asymptotic value $r=1$ (all BR players), and despite the increased information cost. This is a typical effect of strong nonlinearities and indicates that an increase of information cost does not always favor the evolutionary dominance of the LMA heuristic, as one could instead infer. A possible explanation of this phenomenon can be deduced from the time series analysis of Fig. 8. Here, the high information cost leads firms to adopt the LMA heuristic. However, LMA firms have a low production level, indeed very close to zero, while the few operating BR firms produce large output. It follows that this situation is quite profitable for the few BR firms, as prices are high due to low average quantities in the market. As a results, BR firms earn large profits and this generates a sort of "evolutionary frenzy" to switch to the BR heuristic. Once almost all firms are BR, their profits reduce considerably as a consequence of high information costs and low prices, due to high aggregate levels of productions. At this point, the LMA heuristic starts to be the most convenient strategy again, and suddenly LMA firms become the large majority. The cycle goes on until the moment in which the evolutionary thrust towards the BR heuristic is so strong that all firms become BR. From the gray trajectories of Fig. 8, we observe that similar dynamical paths occur even when the oligopoly is forced by random noise. In this case, the sudden jumps from one heuristic to the other are even more prolonged in time and occur more frequently. Moreover, even with random aggregate expenditures on the market, we observed that it never occurs that the LMA heuristic becomes dominant over BR, despite the large cost of the BR heuristic. These results are confirmed further in numerical simulations with memory, which we omit for the sake of brevity. Actually, the higher the memory parameter $\omega$ is, the more pronounced and prolonged in time the phenomenon is. This can be easily explained, since with memory of past profits the enthusiasms for the recent extraordinary positive performances of an heuristic are attenuated by the not-so-extraordinary previous performances: in a sense, firms are "wiser". As for a mathematical explanation, phenomena of on-off intermittency are presumably due to the presence of Milnor attractors, as detected in Cerboni Baiardi et al., 2015 for an evolutionary game with a similar dynamical structure.

\section{Some concluding remarks}

In this paper we have considered an evolutionary game with a population of firms producing homogeneous goods in an oligopolistic market characterized by a nonlinear (isoelastic) demand function with random noise. Firms are subdivided into two subgroups that differ for the adaptive behavioral rule employed to update their productions over time. Both groups are boundedly rational and make use of different amounts of information and computational efforts: a group adopts a Best Reply (BR) strategy with naïve expectations, whereas the other group follows a rougher rule of thumb introduced in Bischi et al. 2007, called Local Monopolistic Approximation (LMA): under LMA, firms use a linear approximation of the demand function and ignore the presence of competitors in the market. Both strategies share the same Nash equilibrium, whose stability conditions are given in terms of the number of firms, players' inertia in updating their productions and the fraction of BR players in the market. In particular, we have proved that an increasing fraction of BR players can cause a reduction of the range of stability of the Nash equilibrium in the parameters space (of course the opposite effect is obtained by increasing the fraction of LMA players). Our results show that even without information costs, an increased presence of players using more information (like those using Best Reply in this model) may reduce stability. However, we have also proved that, for sufficiently high levels of the speed of adjustment, when the Nash equilibrium is stable both under pure BR oligopoly and under pure LMA oligopoly, then its basin of attraction is larger in the presence of all BR firms than with all LMA firms, so in this sense using more information enhances the stability extent of the Nash equilibrium in the state space, provided it is locally stable.

Then the dynamics of the fraction of firms selecting a given behavioral rule are endogenized by introducing evolutionary pressure by means of a profit-driven (exponential) replicator dynamics. We have proved that, assuming suitable information costs associated to the BR strategy, the LMA strategy dominates and all the firms play in the long run the Nash equilibrium

\footnotetext{
${ }^{17}$ For a general treatment of random dynamical systems see Arnold 1998

${ }^{18}$ Simulations are performed using a Monte Carlo approach based on method of antithetic variates, which is one of the most widely used variance reduction technique, see e.g. Boyle et al., 1997
} 

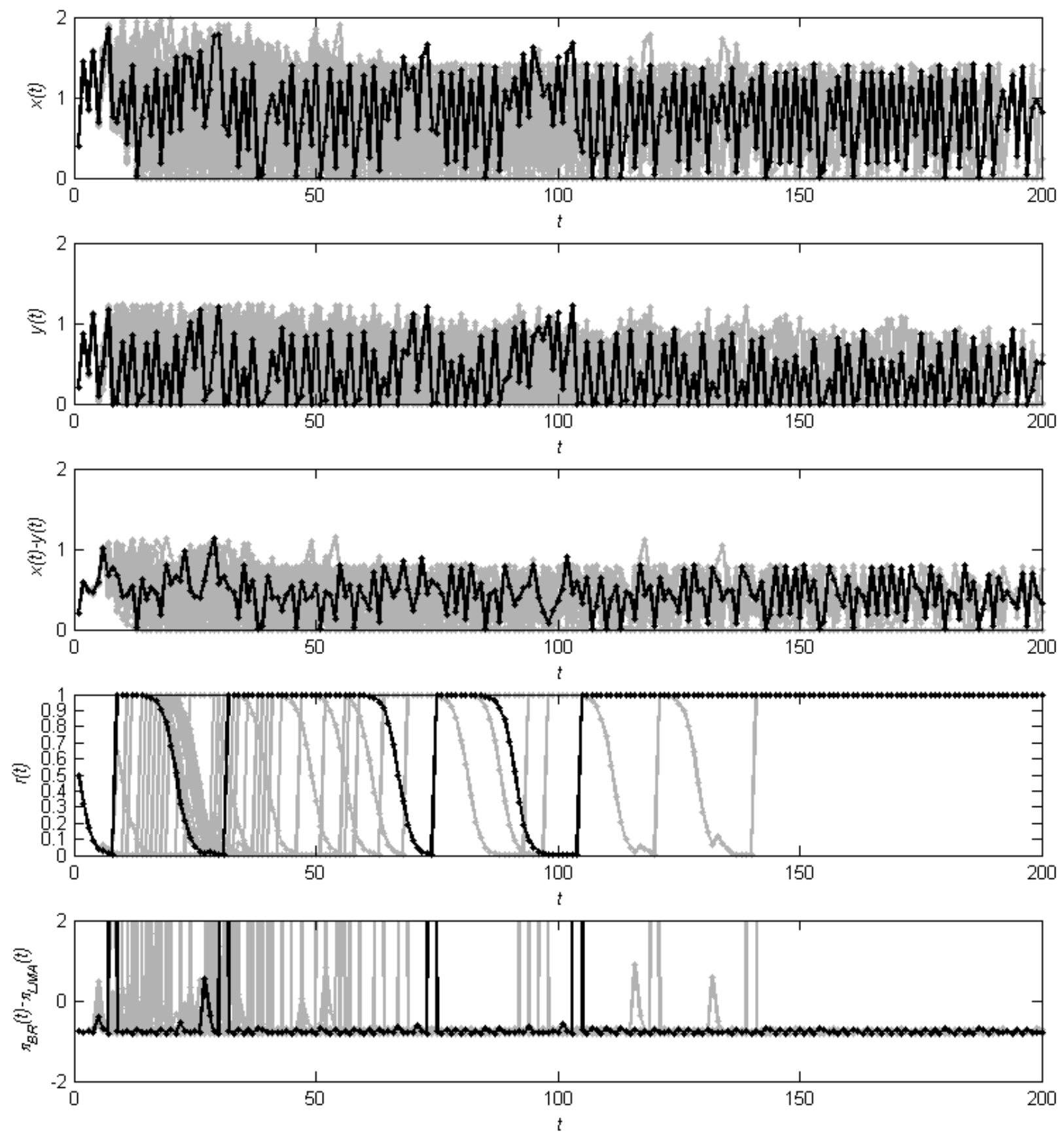

Figure 8: Versus-time sequences of $x(t), y(t), x(t)-y(t), r(t)$ and $\pi_{B R}(t)-\pi_{L M A}(t)$ along a typical trajectory and on-off intermittency phenomena. Parameters as in Fig. 7 a with $K=0.8$. In black $\epsilon=0$. In gray 100 runs for $A(t) \sim \mathcal{U}(1-\epsilon, 1+\epsilon)$, with $\epsilon=0.005$.

whenever it is stable. However, in the case of identical information costs, time evolutions can be obtained leading to long-run coexistence of both BR and LMA behavioral rules, even if the extra information and computational effort to play the BR strategy can be obtained for free. This result may sound quite strange at a first sight, but the main intuition behind it lies in the fact that both strategies are boundedly rational. Thus, the use of more sophisticated computations may be useless when some information is missing, like in the case of naïve BR players. This can occur even if the alternative method requiring 
less information, as LMA considered in this paper, not only employs a linear approximation of the demand function, but also completely ignores the presence of competitors at each step.

More interesting evolutionary situations, both from an economic and a mathematical point of view, are obtained when the Nash equilibrium is unstable, and firms' production choices exhibit oscillatory dynamics (periodic or chaotic) around it. On the basis of analytical and numerical results, we have shown that when an information cost is associated to the BR, then one has to consider the amplitude of the oscillations around the Nash equilibrium $E_{*}$ to assess which behavioral rule survives in the long run: when the oscillations of productions remain inside a given neighborhood of $E_{*}$, i.e. the amplitude of the oscillations does not exceed a given threshold, then the presence of an information cost forces firms to follow the cheaper LMA rule; however, oscillations of larger amplitude induce firms to choose the BR behavioral rule in the long run.

Moreover, in several cases two coexisting attractors exist in the invariant planes $r=0$ and $r=1$, in which all players adopt the same behavioral strategy (LMA and BR respectively). Each attractor has its own basin of attraction, so that the convergence to an attractor where all agents employ the same pure behavioral rule strongly depends on the initial condition of the system, and in particular on the initial fraction $r(0)$. Clearly this implies path dependence and uncertainty about the long-run evolution of the selected behavioral rule. If memory is introduced in the evolutionary process, i.e. agents also consider a discounted average of past profits to update their strategies, then the stability properties of the Nash equilibrium do not change and numerical simulations show that the structure of the attractors of the model remains essentially the same. However the basins of attractions change as memory increases, so that LMA strategy is favored in the long run.

The dynamics of the evolutionary oligopoly are further complicated by the possible absence of an evolutionary dominant heuristic even in presence of positive information and computational costs incurred by BR firms. Indeed, we have shown through numerical simulations the existence of a subregion of the parameters space of the model where information costs for playing the BR heuristic are positive and behavioral heterogeneity arises endogenously as the results of evolutionary competition between the different heuristics.

As a last remark, time patterns have been numerically observed where large oscillations occur between values of the fraction of BR players very close to 0 and values very close to 1 , with a typical phenomenon of on-off intermittency (see e.g. Ashwin et al., 1996. Venkataramani et al. 1996 and Bischi et al., 1998). This is a typical effect of strong nonlinearity and has a clear economic explanation, related to the fact that high information costs lead firms to adopt the LMA heuristic. However, as LMA firms have a low production level while the few operating BR firms produce large output, the situation becomes quite profitable for BR firms, since prices are high due to low average production. As a result, BR firms earn high profits and this generates a sort of "evolutionary frenzy" to switch to the BR heuristic. Once almost all firms are $\mathrm{BR}$, their profits reduces considerably due to the high information costs and low prices, which are a consequence of high aggregate levels of production. At this point, the LMA heuristic returns the most convenient strategy again, and LMA firms become the large majority. This effect is also observed in the presence of random noise, and it is more pronounced and prolonged when memory of past profits is added in the model. Phenomena of on-off intermittency as well as additional global properties of the complete model are surely worth of further investigations.

\section{ACKNOWLEDGMENTS}

We thank an Associate Editor and two Referees for useful comments and suggestions. We also thank the participants to the Oligo Workshop in Rome, June 2014, and to the Sixteenth International Symposium on Dynamic Games and Applications in Amsterdam, July 2014. The usual disclaimer applies. This work has been performed within the framework of COST Action IS1104 "The EU in the new economic complex geography: models, tools and policy evaluation", and under the activities of GNFM, Italy.

\section{Appendix}

Proof of Proposition 1. The results about the existence of the equilibria of the dynamical systems (7) follow from Bischi et al., 2007, while their stability can be studied by standard local analysis as shown in the following. Given the Jacobian matrix of (7) computed at the Nash equilibrium (8)

$$
J\left(E_{*}\right)=\left(\begin{array}{cc}
1-\left(1-r+\frac{N r}{2}\right) \lambda & -\frac{1}{2}(N-2)(1-r) \lambda \\
\alpha r\left(1-\frac{N}{2}\right) & 1-\alpha+\frac{\alpha}{2}(3-N(1-r)-2 r)
\end{array}\right)
$$


a sufficient condition for the local asymptotic stability of $E_{*}$ is that the eigenvalues of $(16)$ are located inside the unit circle of the complex plane. These eigenvalues are the solutions of the characteristic equation $P_{2}(z)=z^{2}-\operatorname{tr} J z+\operatorname{det} J=0$, where

$$
\operatorname{tr} J=2+\frac{\alpha[1-N(1-r)-2 r]-\lambda[2+r(N-2)]}{2}
$$

and

$$
\operatorname{det} J=1+\frac{2 \alpha[1-N(1-r)-2 r]-\lambda[4-2(N-1) \alpha+r(N-2)(2+\alpha)]}{4}
$$

are, respectively, the trace and the determinant of (16). A necessary and sufficient condition for this is given by the following system of inequalities (known as Schur or Jury's conditions, see e.g. Gandolfo, 2010, Elaydi, 1995 and Medio and Lines, 2001)

$$
\left\{\begin{array}{c}
1+\operatorname{tr} J+\operatorname{det} J>0 \\
1-\operatorname{tr} J+\operatorname{det} J>0 \\
1-\operatorname{det} J>0
\end{array}\right.
$$

It is easy to see that the last two inequalities in (17) are always satisfied for all parameters values inside their ranges of variation. Regarding the first inequality, it is satisfied if and only if

$$
2<N<N_{f}
$$

where $N_{f}$ is given in (9). At $N_{f}$ equilibrium (8) loses stability through a flip bifurcation.

Proof of Proposition 3. With $r=0$ (all LMA agents), map (7) becomes

$$
\left\{\begin{array}{c}
\left.x(t+1)=f_{0}(x, y)=\max \left\{0,(1-\lambda) x(t)+\lambda\left[\sqrt{\frac{(N-1) y(t)}{c}}-(N-1) y(t)\right)\right]\right\} \\
y(t+1)=g_{0}(y)=\max \left\{0,(1-\alpha) y(t)+\frac{\alpha}{2}[y(t)+N y(t)(1-c N y(t))]\right\}
\end{array}\right.
$$

where the second component is uncoupled from the first one, i.e. a one-dimensional difference equation (master equation), whereas the first component depends also on the second variable (slave equation). The properties of the one-dimensional map $g_{0}(y)$, which is a quadratic map conjugate to the well known logistic map, strongly influence the dynamics of the whole system. In particular, the positive value of $y$ at which $g_{0}(y)=0$, i.e.

$$
y_{0}=\frac{2+\alpha(N-1)}{\alpha c N^{2}}
$$

gives the boundary of the basin of the attractor, since all trajectories starting with $y(0)>y_{0}$ go to the point $(0,0)$ in one step. Analogously, if $r=1$ (all BR agents), the map (7) becomes

$$
\left\{\begin{array}{l}
\left.x(t+1)=f_{1}(x)=\max \left\{0,(1-\lambda) x(t)+\lambda\left[\sqrt{\frac{(N-1) x(t)}{c}}-(N-1) x(t)\right)\right]\right\} \\
y(t+1)=g_{1}(x, y)=\max \left\{0,(1-\alpha) y(t)+\frac{\alpha}{2}[y(t)+N x(t)(1-c N x(t))]\right\}
\end{array}\right.
$$

where the first component is uncoupled from the second one. In this case, the positive value of $x$ at which $f_{1}(x)=0$, i.e.

$$
x_{1}=\frac{\lambda^{2}(N-1)}{c(\lambda N-1)^{2}}
$$

gives the boundary of the basin of the attractor. Similarly as before, all trajectories starting with $x(0)>x_{1}$ go to the point $(0,0)$ in one step. The assertion of the proposition then follows by straightforward considerations.

Proof of Proposition 4. For any $r \in[0,1]$ and $\omega=0$, at the Nash equilibrium (8) both representative agents produce the same quantity, so from (13) it is straightforward to observe that $\pi_{L M A}-\pi_{B R}=K \geq 0$ according to the assumption on information costs associated to the BR strategy. The expression of the exponential replicator dynamics

$$
\frac{r(t)}{r(t)+(1-r(t)) e^{\beta \Delta \Pi(t)}}=\frac{r(t)}{r(t)+(1-r(t)) e^{\beta\left(\pi_{L M A}(t)-\pi_{B R}(t)\right)}}
$$


at the Nash equilibrium becomes

$$
\frac{r(t)}{r(t)+(1-r(t)) e^{\beta K}}
$$

If $K=0$ then at any point in $E$, it is $\pi_{B R}=\pi_{L M A}$ and, as a consequence, $r(t+1)=r(t)$ for each $t$, So, infinitely many equilibrium points $E_{*}$ of 15 exist, filling the segment $E=\left\{x=\frac{N-1}{c N^{2}}, y=\frac{N-1}{c N^{2}}, 0 \leq r \leq 1\right\}$. Instead, if $K>0$ then at any point in $E$, it is $\pi_{B R}<\pi_{L M A}$, so the stationary condition is only satisfied at the boundary points $E_{0}=\left(\frac{N-1}{c N^{2}}, \frac{N-1}{c N^{2}}, 0\right)$ and $E_{1}=\left(\frac{N-1}{c N^{2}}, \frac{N-1}{c N^{2}}, 1\right)$, located on the invariant planes $r=0$ (all LMA firms) and $r=1$ (all BR firms) respectively.

The local stability properties of these equilibria are determined through the study of the Jacobian matrix computed at the Nash equilibrium:

$$
J\left(\frac{N-1}{c N^{2}}, \frac{N-1}{c N^{2}}, r\right)=\left[\begin{array}{ccc}
1-\left(1-r+\frac{N r}{2}\right) \lambda & -\frac{1}{2}(N-2)(1-r) \lambda & 0 \\
\alpha r\left(1-\frac{N}{2}\right) & 1-\alpha+\frac{\alpha}{2}(3-N(1-r)-2 r) & 0 \\
\frac{r(r-1) \beta c e^{\beta K}}{(N-1)\left(r+(1-r) e^{\beta K}\right)^{2}} & \frac{r(r-1) \beta c e^{\beta K}}{(N-1)\left(r+(1-r) e^{\beta K}\right)^{2}} & \frac{e^{\beta K}}{\left(r+(1-r) e^{\beta K}\right)^{2}}
\end{array}\right]
$$

In the case $K=0$ (zero information cost), we have

$$
J(E)=\left[\begin{array}{ccc}
1-\left(1-r+\frac{N r}{2}\right) \lambda & -\frac{1}{2}(N-2)(1-r) \lambda & 0 \\
\alpha r\left(1-\frac{N}{2}\right) & 1-\alpha+\frac{\alpha}{2}(3-N(1-r)-2 r) & 0 \\
\frac{r(r-1) \beta}{(N-1)} & \frac{r(r-1) \beta}{(N-1)} & 1
\end{array}\right]
$$

hence the characteristic equation becomes $(1-z) P_{2}(z)=0$, where $P_{2}(z)$ is the characteristic polynomial considered in Proposition 1. This implies that $z=1$ is always an eigenvalue (i.e. $E$ are nonhyperbolic equilibria) with corresponding eigenvector along the $(0,0,1)$ direction. Thus, an equilibrium of the segment $E$ characterized by $0 \leq r=r^{*} \leq 1$ is stable if and only if the equilibrium $E_{*}$ of the two-dimensional system (7) with parameter $r=r^{*}$ is stable, see Proposition 1 . From which the first part of the statement follows.

In the case of positive information cost for BR firms $K>0$, we first consider

$$
J\left(E_{0}\right)=\left(\begin{array}{ccc}
1-\lambda & -\frac{\lambda(N-2)}{2} & 0 \\
0 & 1-\alpha+\frac{(3-N) \alpha}{2} & 0 \\
0 & 0 & e^{-\beta K}
\end{array}\right)
$$

which is a triangular matrix; hence, its eigenvalues are given by the diagonal entries: $z_{1}=1-\lambda$ is always in the range $0<z_{1}<1 ; z_{3}=e^{-\beta K}$ is in the range $z_{3} \in\left[e^{-\beta K}, 1\right) \subset(0,1)$ for $\beta>0$. Finally, $z_{2}=1-\alpha+\frac{(3-N) \alpha}{2}$ is inside the stability range $-1<z_{2}<1$ for $\alpha(N-1)<4$, and it crosses the value $z_{2}=-1$ for increasing $\alpha$ or $N$ thus giving rise to a flip (or period-doubling) bifurcation. For a given $\alpha \in(0,1]$, this local bifurcation causing the stability loss of $E_{0}$ occurs when

$$
N_{f}=\frac{4+\alpha}{\alpha}
$$

whereas for a given number of firms $N$, the stability loss occurs when

$$
\alpha_{f}=\frac{4}{N-1}
$$

Moreover, we consider the Jacobian matrix at the Nash equilibrium $E_{1}$ where all firms use BR strategy:

$$
J\left(E_{1}\right)=\left(\begin{array}{ccc}
1-\frac{N}{2} \lambda & 0 & 0 \\
\left(1-\frac{N}{2}\right) \alpha & 1-\frac{\alpha}{2} & 0 \\
0 & 0 & e^{\beta K}
\end{array}\right)
$$

Also in this case we have a triangular matrix, with the eigenvalue $z_{1}=1-\frac{N}{2} \lambda<1$ for all parameters values and $z_{1}>-1$ provided that $N \lambda<4 ; z_{2}$ is always in the range $0<z_{2}<1$ whereas $z_{3}>1$, hence $E_{1}$ is never stable along the direction of the $r$ axis. The second part of the statement follows.

Proof of Proposition 5. For any $r \in[0,1]$, in equilibrium both representative agents produce the same quantity in (8). Thus, from 13 it is straightforward to observe that $\pi_{L M A}-\pi_{B R}=K \geq 0$ according to the assumption on information 
costs associated to the BR strategy. It follows that the condition on the last equation of (15) for having an equilibrium requires $\Delta \Pi=K$. The expression of the exponential replicator dynamics at the Nash equilibrium becomes

$$
\frac{r(t)}{r(t)+(1-r(t)) e^{\beta K}}
$$

If $K=0$ then at any point in $E$, it is $\pi_{B R}=\pi_{L M A}$ and, as a consequence, $r(t+1)=r(t)$ for each $t$. So, infinitely many equilibrium points of 15 exist, filling the segment $E=\left\{x=\frac{N-1}{c N^{2}}, y=\frac{N-1}{c N^{2}}, 0 \leq r \leq 1\right\}$. Instead, if $K>0$ then at any point in $E$, it is $\Delta \Pi>0$, so the stationary condition is only satisfied at the boundary points $E_{0}=\left(\frac{N-1}{c N^{2}}, \frac{N-1}{c N^{2}}, 0, K\right)$ and $E_{1}=\left(\frac{N-1}{c N^{2}}, \frac{N-1}{c N^{2}}, 1, K\right)$, located on the invariant planes $r=0$ (all LMA firms) and $r=1$ (all BR firms) respectively.

The local stability properties of these equilibria are determined through the study of the Jacobian matrix computed at the Nash equilibrium. In the case $K=0$ (zero information cost), the Jacobian has a structure as follows

$$
J(E)=\left(\begin{array}{cccc}
J_{11} & J_{12} & 0 & 0 \\
J_{21} & J_{22} & 0 & 0 \\
J_{31} & J_{32} & J_{33} & 0 \\
J_{41} & J_{42} & J_{43} & \omega
\end{array}\right)
$$

Thus, at $E$ the eigenvalues are the same as in the 3D model (no memory) with the additional eigenvalue $\omega$, which is always in the range $(0,1)$. Thus, the stability conditions are the same and the first part of the statement follows. Moreover, we consider the Jacobian matrix at the Nash equilibrium $E_{0}$ where all firms use LMA strategy, whose structure is

$$
J\left(E_{0}\right)=\left(\begin{array}{cccc}
J_{11} & J_{12} & 0 & 0 \\
0 & J_{22} & 0 & 0 \\
0 & 0 & J_{33} & 0 \\
J_{41} & J_{42} & 0 & \omega
\end{array}\right)
$$

It follows that

$$
\operatorname{det}\left(E_{0}-z\right)=\left(J_{11}-z\right)\left(J_{22}-z\right)\left(J_{33}-z\right)(\omega-z)
$$

The same factorization holds at $E_{1}$ where $r=1$, as in that case in is $J_{12}=0$ and $J_{21} \neq 0$. Thus, at $E_{0}$ and $E_{1}$, the eigenvalues are the same as in the 3D model (no memory) with the additional eigenvalue $\omega$, which is always in the range $(0,1)$. The second part of the statement follows.

\section{References}

Agliari, A, Puu, T, 2002. Oligopoly Dynamics. Models and Tools, chap. : A Cournot duopoly with bounded inverse demand function: Springer Verlag, Berlin.

Agliari, A.A, Gardini, L, Puu, T, 2006. Global bifurcations in duopoly when the Cournot point is destabilized via a subcritical Neimark bifurcation. International Game Theory Review 8, 1-20.

Andronov, A.A, Leontovich, E.A, Gordon, I.I, Maier, A.G, 1967. Theory of Bifurcations of Dinamic Systems on a Plane: Keter Press.

Anufriev, M, Kopnyi, D, Tuinstra, J, 2013. Learning cycles in bertrand competition with differentiated commodities and competing learning rules. Journal of Economic Dynamics \& Control 37, 2562-2581.

Arnold, L, 1998. Random Dynamical Systems: Springer.

Ashwin, P, Buescu, J, Stewart, I, 1996. From attractor to chaotic saddle: a tale of transverse instability. Nonlinearity $9,703-737$.

Bischi, G.I, Chiarella, C, Kopel, M, Szidarovszky, F, 2010. Nonlinear Oligopolies: Stability and Bifurcations: SpringerVerlag, Berlin. 
Bischi, G.I, Gardini, L, Stefanini, L, 1998. Synchronization, intermittency and critical curves in a duopoly game. Mathematics and Computers in Simulation 44, 559-585.

Bischi, G.I, Kopel, M, 2001. Equilibrium selection in a nonlinear duopoly game with adaptive expectations. Journal of Economic Behavior \& Organization 46, 73-100.

Bischi, G.I, Naimzada, A.K, Sbragia, L, 2007. Oligopoly games with local monopolistic approximation. Journal of Economic Behavior \& Organization 62, 371-388.

Boyle, P, Broadie, M, Glasserman, P, 1997. Monte Carlo methods for security pricing. Journal of Economic Dynamic \& Control 21, 1267-1321.

Brock, W, Hommes, C, 1997. A rational route to randomness. Econometrica: Journal of the Econometric Society 65, 10591095.

Cabrales, A, Sobel, J, 1992. On the limit points of discrete selection dynamics. Journal of Economic Theory 57, 407-419.

Cerboni Baiardi, L, Lamanta, F, Radi, D, 2015. Evolution, information and rationality: an example from oligopoly. Submitted .

Dieci, R, Bischi, G, Gardini, L, 2003. Routes to complexity in a business-cycle model described by a noninvertible triangular map. Cubo A Mathematical Journal 5, 367-396.

Dieci, R, Bischi, G.I, Gardini, L, 2001. From bi-stability to chaotic oscillations in a macroeconomic model. Chaos, Solitons \& Fractals 12, 805-822.

Droste, E, Hommes, C.H, Tuinstra, J, 2002. Endogenous fluctuations under evolutionary pressure in Cournot competition. Games and Economic Behavior 40, 232-269.

Elaydi, S.N, 1995. An introduction to difference equations: New York, Springer.

Fanti, L, Gori, L, Sodini, M, 2013. Nonlinear dynamics in a cournot duopoly with different attitudes towards strategic uncertainty. Abstract and Applied Analysis Articol ID 323290.

Fisher, F.M, 1961. The stability of the Cournot oligopoly solution: the effect of speeds of adjustment and increasing marginal costs. The Review of Economic Studies 28, 125-135.

Gandolfo, G, 2010. Economic Dynamics: Springer.

Hahn, F.H, 1962. The stability of the Cournot oligopoly solution. The Review of Economic Studies 29, 329-331.

Hofbauer, J, Sigmund, K, 2003. Evolutionary game dynamics. Bulletin (New Series) of the American Mathematical Society $40,479-519$.

Hofbauer, J, Weibull, J.W, 1996. Evolutionary selection against dominated strategies. Journal of Economic Theory 71, 558573.

Hommes, C, 2013. Behavioral rationality and heterogeneous expectations in complex economic systems: Cambridge University Press.

Hommes, C, Kiseleva, T, Kuznetsov, Y, Verbic, M, 2012. Is more memory in evoluionary selection (de)stabilizing? Macroeconomic Dynamics 16, 335-357.

Hommes, C.H, Ochea, M.I, Tuinstra, J, 2011. On the stability of the Cournot equilibrium: An evolutionary approach. Working paper.

Huyck, J.B.V, Battalio, R.C, Beil, R.O, 1990. Tacit coordination games, strategic uncertainty, and coordination failure. The American Economic Review 80, 234-248.

Huyck, J.B.V, Cook, J.P, Battalio, R.C, 1994. Selection dynamics, asymptotic stability, and adaptive behavior. Journal of Political Economy 102, 975-1005. 
Huyck, J.B.V, Cook, J.P, Battalio, R.C, 1997. Adaptive behavior and coordination failure. Journal of Economic Behavior \& Organization 32, 483-503.

Kolyada, S.F, 1992. On dynamics of triangular maps of the square. Ergodic Theory and Dynamical Systems 12, 749-768.

Kopel, M, Lamantia, F, Szidarovszky, F, 2014. Evolutionary competition in a mixed market with socially concerned firms. Journal of Economic Dynamics \& Control 48, 394-409.

Kuznetsov, Y.A, 1998. Elements of Applied Bifurcation Theory: Springer.

Lamantia, F, 2011. A nonlinear duopoly with efficient production-capacity levels. Computational Economics 38, 295-309.

Lambertini, L, 2010. Oligopoly with hyperbolic demand: A differential game approach. Journal of Optimization Theory and Applications 145, 108-119.

McManus, M, Quandt, R, 1961. Comments on the stability of the Cournot oligipoly model. The Review of Economic Studies 28, 136-139.

Medio, A, Lines, M, 2001. Nonlinear dynamics: Cambridge University Press.

Puu, T, 1991. Chaos in duopoly pricing. Chaos, Solitons \& Fractals 1, 573-581.

Theocharis, R.D, 1960. On the stability of the Cournot solution on the oligopoly problem. Review of Economic Studies $27,133-134$.

Tramontana, F, Gardini, L, Puu, T, 2010. Global bifurcations in a piecewise-smooth Cournot duopoly game. Chaos, Solitons \& Fractals 43, 15-24.

Tuinstra, J, 2004. A price adjustment process in a model of monopolistic competition. International Game Theory Review $6,417-442$.

Vallée, T, Yildizoglu, M, 2009. Convergence in the finite Cournot oligipoly with social and individual learning. Journal of Economic Behavior \& Organization 72, 670-690.

Vega-Redondo,, 1997. The evolution of walrasian behavior. Econometrica: Journal of the Econometric Society 65, 375-384.

Venkataramani, S.C, Hunt, B.R, Ott, E, 1996. Bubbling transition. Physical Reiew E 54, 1346-1360.

Vriend, N.J, 2000. An illustration of the essential di!erence between individual and social learning, and its consequences for computational analyses. Journal of economic dynamics \& control 24, 1-19. 